\title{
Entropy functionals and thermodynamics of relativistic geometric flows, stationary quasi-periodic Ricci solitons, and gravity
}

\author{
Iuliana Bubuianu* \\ Radio Iaşi, 44 Lască Catargi, Iaşi, 700107, Romania \\ Sergiu I. Vacaru ${ }^{\dagger}$ \\ Physics Department, California State University at Fresno, Fresno, CA 93740, USA; and \\ Dep. Theoretical Physics and Computer Modelling, 101 Storozhynetska street, Chernivtsi, 58029, Ukraine \\ and \\ Elşen Veli Veliev ${ }^{\ddagger}$ \\ Department of Physics, Kocaeli University, 41380, Izmit, Turkey
}

October 7, 2020

\begin{abstract}
We investigate gravity models emerging from nonholonomic (subjected to non-integrable constraints) Ricci flows. Considering generalizations of G. Perelman's entropy functionals, relativistic geometric flow equations, nonholonomic Ricci soliton and equivalent (modified) Einstein equations are derived. There are studied nonholonomic configurations which allow explicit modeling of entropic scenarios for gravity and dark matter (in the E. Verlinde approach and/or other variants). It is shown that using the anholonomic frame deformation method, the systems of nonlinear partial differential equations for geometric flow evolution of nonlinear stationary gravitations systems can be decoupled and integrated in general forms. In this and a series of partner works, we elaborate on stationary models of emergent gravity with quasi-periodic gravitational, matter fields and dark energy/matter structure. Such configurations cannot be described thermodynamically using the concept of Bekenstein-Hawking entropy if area-entropy, holographic or duality relations are not involved. Nevertheless, generalizing G. Perelman statistic thermodynamic approach to models of relativistic Ricci flows and nonholonomic solitons, we can compute respective thermodynamic variables for all types of gravitational and matter field configurations and their geometric evolution. Nonholonomic deformations of the F- and W-entropy considered and relativistic thermodynamic models are studied in more general cases when physically important solutions with quasi-periodic and pattern forming structure are found in modified gravity theories (MGT) and general relativity (GR).
\end{abstract}

Keywords: Relativistic geometric flows; generalized Perelman F- and W-entropy; gravity and nonholonomic Ricci solitons; geometric thermodynamics; off-diagonal solutions with quasi-periodic structure.

PACS2010: 02.40.-k, 02.90. + p, 04.20Cv, 04.20.Jb, 04.50.-h, 04.90. $+\mathrm{e}, 05.90 .+\mathrm{m}$

MSC2010: 53C44, 53C50, 53C80, 82D99, 83C15, 83C55, 83C99, 83D99, 35Q75, 37J60, 37D35

\footnotetext{
*email: iulia.bubu@gmail.com

${ }^{\dagger}$ emails: sergiu.vacaru@gmail.com and sergiuvacaru@mail.fresnostate.edu ; Address for post correspondence in 2019-2020 as a visitor senior researcher at YF CNU Ukraine is: 37 Yu. Gagarina street, ap. 3, Chernivtsi, Ukraine, 58008

${ }^{\ddagger}$ email: elsen@kocaeli.edu.tr and elsenveli@hotmail.com
} 


\section{Contents}

1 Introduction

2 Nonholonomic F- and W-functionals \& geometric flow equations 4

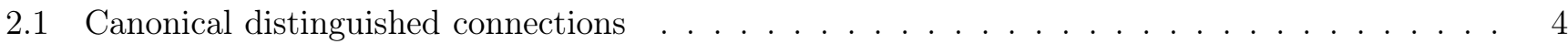

2.2 Modified canonical Perelman's functionals and R. Hamilton equations . . . . . . . . . . . 5

2.3 Nonholonomic Ricci solitons and (modified) Einstein equations . . . . . . . . . . . . . . 6

3 Geometric flow evolution to stationary quasi-periodic structures 8

3.1 Stationary quasicrystal spacetime and effective matter configurations . . . . . . . . . . . 9

3.2 Nonlinear PDEs for the geometric flow evolution of stationary quasi-periodic configurations . 10

4 Computing Perelman thermodynamic variables instead of Bekenstein-Hawking ones 12

4.1 Geometric thermodynamics for relativistic nonholonomic Ricci flows . . . . . . . . . . . . 12

4.2 Computing Perelman's thermodynamic variables for stationary quasi-periodic geometric flows 13

4.2.1 Fixing normalization and integration functions and effective cosmological constants . . 13

4.2.2 Thermodynamic variables for stationary quasi-periodic generating functions \& sources 15

4.2.3 Perelman's thermodynamics for BHs deformed by stationary quasi-periodic structures 15

4.2.4 Thermodynamics of small parametric stationary quasi-periodic BH deformations . . . 16

\section{Conclusion}

A $\mathbf{2}+\mathbf{2}$ and 3+1 N-adapted Variables and Quasi-Periodic Structures

A.1 Geometry of Lorentz manifolds with nonholonomic $2+2$ splitting . . . . . . . . . . . . . . 18

A.2 Nonholonomic distributions with quasiperiodic and/or pattern forming structures . . . . . . 19

\section{Introduction}

The Einstein equations can be derived in thermodynamic models with area-entropy for horizons of black holes, BH, and/or, for instance, making certain assumptions on elastic properties of gravity [1, 2, 3]. Using entropy functionals for geometric flows, the gravitational field equations are derived as self-similar configurations, i.e. (modified) Ricci solitons [4], for Riemannian metrics. Various physical models for noncommutative, relativistic geometric thermodynamic, supersymmetric and modified flow evolution and gravity where elaborated in [5, 6, 7, 8] and references therein, and in quantum information geometric flow theory [9]. This supports the idea and indication of the emergent nature of spacetime when gravity comes from the laws of BH thermodynamics [10, 11, 12, 13] or other above mentioned types of statistical/ phenomenological / geometric thermodynamical theories. In the past four decades, the Bekenstein-Hawking entropy and Hawking temperature played the central role for research in various directions of modern gravity, cosmology and astrophysics. Significant advances started with the explanation of the microscopic origin of Bekenstein-Hawking entropy in string theory [14]; with a subsequent development of the (anti) de Sitter, (A)dS black hole physics and/or cosmology, conformal field theories, CFTs, and AdS/CFT, correspondence [15]; and formulating quasilocal relations when analogous laws of the thermodynamics are defined for various sorts of 'apparent' horizons [16]. Latter, it was realized that similar formulas characterize quantum entanglement [17] and explain the connectivity of the classical spacetime [18]. Recent theoretical advances reveal a deep connection between the quantum information theory and ideas on emergence of spacetime and gravity, for instance, by deriving the (linearized) Einstein equations from general quantum information principles [19, 20, 21, 22, 23, 24.

E. Verlinde conjectured [3, 24] that gravity links to an entropic force as a spacetime elasticity when the gravitational interactions would result from the information regarding the positions of material bodies. His 
models entail the hypothesis that gravity should be viewed as an emergent phenomenon. It joins a thermal gravity-treatment to 't Hooft's and many other authors' efforts involving the holographic principle in particle physics, quantum gravity, cosmological inflation, cosmology, dark energy and dark matter physics etc. The literature on such directions of modern theoretical and mathematical physics and information theory is immense, see [25, 26, 27, 28, 29, 9] and references therein. Nevertheless, this paper deals with none of these issues, though. We just show that well known and fundamental geometric and classical physics reasoning support certain ideas on the origin of gravity as an effect of the entropic force. We argue also that this can be grounded and explained in a rigorous mathematical form using relativistic (and certain noncommutative, nonholonomic, supersymmetric etc.) generalizations of the theory of Ricci flow evolution [5, 6, 7, 8, 9].

It should be noted here that E. Verlinde works [3, 24] and a covariant formulation due to S. Hossenfelder [30] were criticized by authors of [31, 32]. Perhaps, certain technical and theoretical inconsistencies are present in all these works which are consequences of the model dependence and attempts to explain observational data with periodic up-dates and new information, for instance, on dark matter physics. Nevertheless, we consider that the most important ideas and explicit constructions of models of emergent gravity and spacetime thermodynamics have a rigorous support from the theory of geometric flow evolution and geometric methods of constructing exact solutions for systems of nonlinear partial differential equations, PDEs.

The main goal of this work (see details, proofs, more examples and applications in a series of partner articles [9, 33, 34, 35]) is to prove that E. Verlinde conjecture that gravity can be derived from an entropic force and related spacetime elasticity can be considered as a modified relativistic version of the Poincaré conjecture when the G. Perelman W-entropy can be used for deriving both gravitational equations and their thermodynamic properties. For certain well-defined conditions on geometric flows of Riemannian and Kähler metrics, the Poincaré-Thurston conjecture was proven in a rigorous mathematical form 44, see also some early works [36, 37, 38, and reviews of geometric results in [39, 40, 41, 42]. There were not elaborated such rigorous analytic, geometric and topological tools, for instance, for pseudo-Riemannian spaces, thermodynamic geometry and almost Kähler manifolds which are important for applications in classical and quantum gravity and modern cosmology and astrophysics. These are tasks for future research in modern geometry and mathematical physics. Nevertheless, using the anholonomic frame deformation method, AFDM, see a review of results in 43] (on MGTs and applications in modern cosmology, see [44, [45, 46]), it is possible to construct explicit solutions for important nonlinear systems of PDEs describing the statistical thermodynamics and kinetics of spacetime geometric flows and emergent gravity. Such methods and results are well-defined mathematically. In a series of our works [5, 6, 7, 8] (see also references therein), we elaborated on applications in classical and quantum physics of certain geometric methods related to entropy type functionals.

This paper is organized as follows: We begin section 2 with some geometric preliminaries on nonlinear and distinguished connection geometry on nonholonomic Lorentz manifolds. Then we define the nonholonomic canonical version of G. Perelman F-functional and W-functional (equivalently, W-entropy) and speculate how relativistic geometric flow (generalized R. Hamilton) equations can be derived from such functionals. It is argued that the (modified) Einstein equations are certain subclasses of nonholonomic Ricci solitons consisting self-similar configurations with a fixed geometric evolution parameter. In section 3, we study the system of nonlinear PDEs for geometric flow evolution of stationary quasi-periodic gravitational and (effective) matter field configurations. The general decoupling and integration properties of such equations and their respective nonlinear symmetries are analyzed following the AFDM. Section 4 is devoted to the thermodynamics of stationary quasi-periodic geometric flows. We conclude by showing why the nonholonomic relativistic generalizations of G. Perelman's thermodynamics must be considered instead of the well-known Bekenstein-Hawking thermodynamics due to the fact that the most general solutions of such nonlinear PDEs are not characterized in terms of entropy-area values. We provide explicit examples how to compute such geometric quasi-periodic flow thermodynamic variables. Conclusions and discussions are presented in section 5 . 


\section{Nonholonomic F- and W-functionals \& geometric flow equations}

Let us consider a (pseudo) Riemannian manifold $\mathbf{V}$ of dimension $n+m$, with $n, m \geq 2$ (for GR and four dimensional, 4-d, modifications, we can consider $\operatorname{dim} V=4=2+2$ ). Such a manifold is nonholonomic if it is endowed with a non integrable $(n+m)$ splitting of dimensions into conventional horizontal, h, and vertical, $\mathrm{v}$, components defined by a Whitney sum

$$
\mathbf{N}: T \mathbf{V}=h \mathbf{V} \oplus v \mathbf{V}
$$

where $T \mathbf{V}$ is the tangent bundle on $\mathbf{V}$. In 4-d, we can consider a Lorentzian manifold with local pseudoEuclidean signature $(+++-)$ for a metric field $\mathbf{g}=(h \mathbf{g}, v \mathbf{g})$. Any nonlinear connection, N-connection, structure $\mathbf{N}$ defines corresponding subclasses of N-adapted (co) frames which allows, for instance, nonholonomic diadic decompositions of geometric and physical objects. Such N-adapted decompositions can be computed for a corresponding set of coefficients $N_{i}^{a}$, when $\mathbf{N}=N_{i}^{a}(u) d x^{i} \otimes \partial_{a}$. Details on geometric constructions in abstract and coefficient forms in theories with nonholonomic spacetime geometry are given in [43, 33, 34, 35, 5, 6, 7, 8, and references therein 1 ,

\subsection{Canonical distinguished connections}

There are two important linear connections determined by the same metric structure:

$$
\mathbf{g} \rightarrow\left\{\begin{array}{ccc}
\nabla: & \nabla \mathbf{g}=0 ; \quad \nabla \mathbf{T}=0, \quad \text { the Levi-Civita, LC, connection; } \\
\mathbf{D}: & \mathbf{D} \mathbf{g}=0 ; h \mathbf{T}=0, v \mathbf{T}=0 . \quad \text { the canonical d-connection }
\end{array}\right.
$$

The LC-connection $\nabla$ can be introduced without any $\mathrm{N}$-connection structure but it can be always distorted to a necessary type distinguished connection (d-connection) preserving under parallelism the decomposition $\mathbf{N}$. In our previous works, we wrote $\widehat{\mathbf{D}}$ for the canonical d-connection in (1). In this paper, we shall omit "hats" because all constructions will be performed only for the canonical d-connection subjected to such a canonical distortion relation, $\mathbf{D}[\mathbf{g}, \mathbf{N}]=\nabla[\mathbf{g}, \mathbf{N}]+\mathbf{Z}[\mathbf{g}, \mathbf{N}]$. In these formulas, $\mathbf{Z}$ is the distortion tensor determined in standard algebraic form by the torsion tensor $\mathbf{T}[\mathbf{g}, \mathbf{N}]$ of $\mathbf{D}$; both values are completely defined by the metric tensor $\mathbf{g}$ adapted to $\mathbf{N}$ as in [43, 5, 6, 7, 8, The values $h \mathbf{T}$ and $v \mathbf{T}$ are respective torsion components which vanish on conventional $\mathrm{h}$ - and $\mathrm{v}$-subspaces, but there are nontrivial components $h v \mathbf{T}$ defined by certain anholonomy (equivalently, nonholonomic/non-integrable) relations. This means that with respect to certain $\mathrm{N}$-adapted bases we obtain zero values for the torsion coefficients with pure h- and/or vindices and non-zero values for the torsion components with mixed $\mathrm{h}$ - and v-indices, depending on off-diagonal coefficients of metrics and respective anholonomy coefficients of a N-connection structure.

All geometric constructions on $\mathbf{V}$ can be performed equivalently with $\nabla$ (in a not adapted form to $\mathbf{N}$ ) and/or in a form adapted to the N-connections splitting using, for instance, $\mathbf{D}$ from (1). The N-adapted coefficients for a canonical d-connection $\mathbf{D}=(h \mathbf{D}, v \mathbf{D})$ and, corresponding torsion, $\mathbf{T}_{\alpha \beta}^{\gamma}$, Ricci tensor, $\mathbf{R}_{\beta \gamma}$, scalar curvature ${ }^{s} R:=\mathbf{g}^{\alpha \beta} \mathbf{R}_{\beta \gamma}$, and Einstein tensor, $\mathbf{E}_{\beta \gamma}:=\mathbf{R}_{\beta \gamma}-\frac{1}{2} \mathbf{g}_{\beta \gamma}{ }^{s} R$, are defined and computed in standard forms and related via distortion formulas to respective values determined by $\nabla$. Thus, any (pseudo) Riemannian geometry and gravity theory, and various metric-affine modifications (for instance, $F(R)$-modified theories [44, 43]), can be formulated equivalently using geometric data (g, $\nabla)$ and/or (g, D). The priority of the nonholonomic canonical variables $(\mathbf{g}, \mathbf{D})$ is that they allow to decouple and integrate in some very general form various types of modified and standard Einstein equations for generic off-diagonal metrics with

\footnotetext{
${ }^{1}$ For a local calculus on $V$, we can parameterize the coordinates in the form: $u^{\mu}=\left(x^{i}, y^{a}\right)$, (in brief, $u=(x, y)$ ), where indices respectively take values of type $i, j, \ldots=1,2 \ldots, n$ and $a, b, \ldots=n+1, n+2, \ldots, n+m$. In this and the partner work 33, 34, 35, the small Greek indices take typical values $\alpha, \beta, \ldots=1,2, n+m$. In 4 -d, we consider that $n=m=2$ and $u^{4}=y^{4}=t$ is the time like coordinate; we follow the Einstein convention on summation on "up-low" repeating indices and use boldface symbols for spaces and geometric objects adapted to a N-connection splitting.
} 
coefficients depending on all spacetime coordinates and for large classes of generating and integration functions and (effective) generating sources. We cite our recent article [43] and references therein for a review in the so-called anholonomic frame deformation method, AFDM, of constructing exact solutions in GR and MGTs, geometric flow theory, and applications in modern cosmology and astrophysics.

We also note that any nonholonomic 4-d manifold $\mathbf{V}$ can be enabled with a double nonholonomic $2+2$ and 3+1 splitting [6, 7, 8, which is important both for constructing new classes of generic off-diagonal exact solutions of nonlinear geometric flow evolution and/or dynamical equations and, respectively, defining and computing associated statistical / quantum / geometric thermodynamic variables.

To study geometric flows we consider a family of metrics $\mathbf{g}(\tau)=\mathbf{g}(\tau, u)$ and $\mathrm{N}$-connections $\mathbf{N}(\tau)=$ $\mathbf{N}(\tau, u)$ canonically defining respective $\mathbf{D}(\tau)=\mathbf{D}(\tau, u)$, all parameterized by a positive parameter $\tau, 0 \leq \tau \leq$ $\tau_{0}$. Writing down various formulas, it will be usually emphasized the dependence on $\tau$ (without coordinates $u=u^{\alpha}$ ) if that will not result in ambiguities. We also suppose that on $\mathbf{V}$ there are defined families of Lagrange densities ${ }^{g} \mathcal{L}(\tau)$, for gravitational fields in a MGT or GR, and ${ }^{t o t} \mathcal{L}(\tau)$, as total Lagrangians for effective and matter fields which will be defined below (an example will be given by formulas (66)). For a double $2+2$ and $3+1$ splitting, we can consider local coordinates labeled as $u^{\alpha}=\left(x^{i}, y^{a}\right)=\left(x^{\imath}, u^{4}=t\right)$ for $i, j, k, \ldots=1,2 ; a, b, c, \ldots=3,4$; and $\grave{i}, \grave{j}, \grave{k}=1,2,3$. The nonholonomic distributions for N-connections can be parameterized always in such forms that any open region $U \subset \mathbf{V}$ is covered by a family of 3-d spacelike hypersurfaces $\Xi_{t}$ with a time like parameter $t$.

\subsection{Modified canonical Perelman's functionals and R. Hamilton equations}

For families of variables $(\mathbf{g}(\tau), \mathbf{D}(\tau))$ defining geometric evolution scenarios of 4-d nonholonomic Lorentz manifolds enabled with distributions of Lagrange densities ${ }^{g} \mathcal{L}(\tau)=F\left({ }^{s} R\right)$ and ${ }^{t o t} \mathcal{L}(\tau)$, the modified Perelman's functionals are postulated in the form

$$
\mathcal{F}(\tau)=\int_{t_{1}}^{t_{2}} \int_{\Xi_{t}} e^{-f} \sqrt{|\mathbf{g}|} d^{4} u\left[F\left({ }^{s} R\right)+{ }^{t o t} \mathcal{L}+|\mathbf{D} f|^{2}\right]
$$

and

$$
\mathcal{W}(\tau)=\int_{t_{1}}^{t_{2}} \int_{\Xi_{t}}(4 \pi \tau)^{-3} e^{-f} \sqrt{|\mathbf{g}|} d^{4} u\left[\tau\left(F\left({ }^{s} R\right)+{ }^{t o t} \mathcal{L}+|h \mathbf{D} f|+|v \mathbf{D} f|\right)^{2}+f-8\right],
$$

where the condition $\int_{t_{1}}^{t_{2}} \int_{\Xi_{t}}(4 \pi \tau)^{-3} e^{-f} \sqrt{|\mathbf{g}|} d^{4} u=1$ is imposed on the normalizing function $f(\tau, u)$. The difference from the original Grisha Perelman F- and W-functionals [4] introduced for the Ricci flows of 3-d Riemannian metrics (see details in monographs [39, 40, 41]) is that we study geometric flows of canonical geometric data $(\mathbf{g}(\tau), \mathbf{N}(\tau), \mathbf{D}(\tau))$ for nonholonomic Lorentz manifolds and various generalizations for MGTs as in [43, 5, 6, 7, 8]. In formulas (2) and (3), we consider the gravitational Lagrangian ${ }^{g} \mathcal{L}=F\left({ }^{s} R\right)$ as a functional of the scalar curvature for $\mathbf{D}$, or ${ }^{g} \mathcal{L}=R[\nabla]$ for considering as particular cases models of geometric evolution of exact solutions in GR.

Functionals of type $\mathcal{F}$ and $\mathcal{W}$ were considered in our works for deriving relativistic nonlinear flow evolution equations and encoding modified gravity analogs of the Hamilton equations studied in geometric analysis and topology [38. It should be noted here similar geometric flow equations related to quantum renormalization group equations were considered in physical literature [36, 37] some years before classical mathematical works due to $\mathrm{R}$. Hamilton. The functional $\mathcal{W}$ transforms into the standard Perelman W-entropy [4] (being analogous to minus entropy) for non-relativistic holonomic flows of 3-d hypersurface Riemannian metrics. It was considered as an entropy type value for formulating a statistical thermodynamics model for Ricci flows. In this and partner [33, 34, 35] papers, we work with generalized entropy functionals determined by $F\left({ }^{s} R\right)+{ }^{t o t} \mathcal{L}$ and $\mathbf{D}$, in modified gravity theories, MGTs, and/or by a pseudo-Riemannian $R$ and $\nabla$, in general relativity, GR, instead of standard Riemannian or Kähler configurations used in former standard mathematical works. In our approach, above $\mathrm{F}$ - and $\mathrm{W}$-functionals characterize relativistic thermodynamic models with analogous 
nonlinear hydrodynamic flows of families of entropic variables, metrics and generalized connections, encoding interactions of gravitational and matter fields as it is motivated in [6, 7, 8]. We can compute relativistic entropies (2) and (3) for any $3+1$ splitting with 3 -d closed hypersurface fibrations $\widehat{\Xi}_{t}$. In general, it is possible to work with any class of normalizing functions $f(\tau, u)$ which can be fixed by certain constant values or conditions simplifying some systems of nonlinear PDEs. In many cases, such a function is chosen in a nonexplicit form which allows us to study non-normalized geometric flows but with nonholonomic constraints which allow general decoupling and integration of respective physically important systems of nonlinear PDEs. Such generic off-diagonal solutions can be constructed in explicit form [33, 34, 35, 43, 5, 6, 7, 8, which validates our nonholonomic geometric flow entropic approach, involving metrics with pseudo-Euclidean signature even analogs of Poincaré-Thurston conjecture have not been formulated and proven for the Lorentzian spacetimes.

Following a N-adapted variational procedure, for instance, for the functional $\mathcal{F}(\tau)$ (2) (see details in [6, 17, 8, 33, 34, 35] being, in principle, similar rigorous mathematical proofs in 4, 39, 40, 41, 42, 2), we obtain a system of nonlinear PDEs that generalize the R. Hamilton equations in order to perform an evolution if canonical data $\left(\mathbf{g}=\left\{\mathbf{g}_{\mu \nu}=\left[g_{i j}, g_{a b}\right]\right\}, \mathbf{N}=\left\{N_{i}^{a}\right\}, \mathbf{D},{ }^{t o t} \mathcal{L}\right)$ via a nonholonomic geometric flow:

$$
\begin{aligned}
\partial_{\tau} g_{i j} & =-2\left(\mathbf{R}_{i j}-{ }^{t o t} \Upsilon_{i j}\right) ; \partial_{\tau} g_{a b}=-2\left(\mathbf{R}_{a b}-{ }^{t o t} \Upsilon_{a b}\right) \\
\mathbf{R}_{i a} & =\mathbf{R}_{a i}=0 ; \mathbf{R}_{i j}=\mathbf{R}_{j i} ; \mathbf{R}_{a b}=\mathbf{R}_{b a} \\
\partial_{\tau} f & =-\widehat{\square} f+|\mathbf{D} f|^{2}-{ }^{s} R+{ }^{t o t} \Upsilon_{\alpha}^{\alpha}
\end{aligned}
$$

where $\hat{\square}=\mathbf{D}^{\alpha} \mathbf{D}_{\alpha}$ and ${ }^{t o t} \Upsilon_{\alpha \beta}$ is defined in next section (see formulas (7D)). The conditions $R_{i a}=0$ and $R_{a i}=0$ for the Ricci tensor $\operatorname{Ric}[\mathbf{D}]=\left\{\mathbf{R}_{\alpha \beta}=\left[R_{i j}, R_{i a}, R_{a i}, R_{a b},\right]\right\}$ are necessary if we want to keep the metric $\mathbf{g}(\tau)$ to be symmetric under nonholonomic Ricci flow evolution. We note that similar variational and/or geometric methods allows to derive from $\mathcal{W}(\tau)$ (3) another types nonlinear evolution equations which are equivalent to (4).

This work is on defining entropy functionals and thermodynamic variables for relativistic geometric flows. Formulas (2) and (31) posses entropy properties for metrics of Euclinean signatures and (along causal curves) for metrics of pseudo-Euclidean signature for a respective nonholonomic $3+1$ splitting. Such geometric thermodynamic objects can be computed by integrating on respective measures both for relativistic and non relativistic flow scenarios even the solutions of (generalized) Hamilton equations (4) may result in appearance of singularities (like neck-neck-pinch, cusps, etc. This consists very subtle points on (relativistic) geometric analysis and topology, when Perelman's approach is relied crucially on Ricci flows with surgery, i.e. removal of singularities by caps. The goals of this article are not so general (for Riemannian metrics, there were necessary almost a hundred years [4, 38] with proofs on hundreds of pages [39, 40, 41, 42], in order to prove the Poincaré-Thurston conjecture and it is not clear if, how, and how long it will take to formulate and prove certain relativistic generalizations of such a conjecture). Applying the AFDM, we decouple and find general classes of generic off-diagonal solutions of relativistic flow equations and, in particular, for (generalized) Einstein equations with quasiperiodic structure. Such solutions cannot be characterized by BekensteinHawking thermodynamic variables but using Perelman's like thermodynamic models based on (3) we can elaborate on classical and quantum information flow models and applications in modern gravity, cosmology and astrophysics [6, 7, 9, 47, 33, 34, 35].

\subsection{Nonholonomic Ricci solitons and (modified) Einstein equations}

A Ricci soliton is a self-similar solution to the Ricci flow equations (for Riemannian metrics. Such configurations homothetically strink, remain steady or expand under geometric flow evolution, see details in [4, 38, 39, 40, 41, 42]), and can be respectively studied for a fixed point $\tau=\tau_{0}$. Considering nonlinear systems with $\partial_{\tau} \mathbf{g}_{\mu \nu}=0$ and for a specific choice of the normalizing geometric flow function $f$, the equations

\footnotetext{
${ }^{2}$ in our case, we use $\mathbf{D}$ instead of $\nabla$ and the so-called $\mathrm{N}$-adapted differential and partial derivatives
} 
(44) transform into relativistic nonholonomic Ricci soliton equations. Such systems of nonlinear PDEs are equivalent to (modified) Einstein equations in (MGT) GR for corresponding definitions of effective sources of ${ }^{t o t} \Upsilon_{\alpha \beta}$,

$$
\begin{aligned}
\mathbf{R}_{i j} & ={ }^{t o t} \Upsilon_{i j}, \\
\mathbf{R}_{a b} & ={ }^{t o t} \Upsilon_{a b}, \\
\mathbf{R}_{i a} & =\mathbf{R}_{a i}=0 ; \mathbf{R}_{i j}=\mathbf{R}_{j i} ; \mathbf{R}_{a b}=\mathbf{R}_{b a} .
\end{aligned}
$$

A class of MGTs and GR can be formulated as geometric models of entropic elasticity which is similar to the idea of emergent gravity put forward by E. Verlinde [3, 24, or modelled as different types of (massive) metric-affine, Finsler generalized and other type MGTs, see details in [33, 34, 35, 43, 5, 6, 7, 8]. In this work, we do not elaborate on an explicit model of "elastic gravity" and possible applications in dark energy and dark matter physics but show that such theories can be derived as certain examples of relativistic geometric flows prescribing respective effective Lagrangians for W-entropy and relate sources in generalized Hamilton equations (4). More than that, our constructions are motivated by the ideas that MGTs and GR can be generated as thermodynamic models but following a rigorous geometric flow approach with a relativistically generalized G. Perelman thermodynamics, see below section 4.1 .

Let us study the conditions when entropic elastic scenarios can be derived from nonholonomic Ricci solitons. We introduce three important values determined by a conventional displacement vector field $\mathbf{u}^{\alpha}$, cosmological constant $\Lambda$ and some constants $\alpha, \beta, \gamma$ :

$$
\begin{aligned}
\varepsilon_{\alpha \beta} & =\mathbf{D}_{\alpha} \mathbf{u}_{\beta}-\mathbf{D}_{\beta} \mathbf{u}_{\alpha} \text { - the elastic strain tensor ; } \phi=u / \sqrt{|\Lambda|} \text { - a dimensionless scalar } ; \\
\chi & =\alpha\left(\mathbf{D}_{\mu} \mathbf{u}^{\mu}\right)\left(\mathbf{D}_{\nu} \mathbf{u}^{\nu}\right)+\beta\left(\mathbf{D}_{\mu} \mathbf{u}_{\nu}\right)\left(\mathbf{D}^{\mu} \mathbf{u}^{\nu}\right)+\gamma\left(\mathbf{D}_{\mu} \mathbf{u}_{\nu}\right)\left(\mathbf{D}^{\nu} \mathbf{u}^{\mu}\right) \text { - a general kinetic term for } \mathbf{u}^{\mu},
\end{aligned}
$$

when short hands $u:=\sqrt{\left|\mathbf{u}_{\alpha} \mathbf{u}^{\alpha}\right|}, \varepsilon=\varepsilon_{\beta}^{\beta}$, and $\mathbf{n}^{\alpha}:=\mathbf{u}^{\alpha} / u$ are used. For (2) and (3), and respective (41), there are considered nonholonomic distributions when corresponding total, effective gravitational, usual matter, interaction and kinetic terms Lagrangians are postulated in the form ${ }^{t o t} \mathcal{L}={ }^{g} \mathcal{L}+{ }^{m} \mathcal{L}+{ }^{i n t} \mathcal{L}+{ }^{\chi} \mathcal{L}$ for

$$
{ }^{g} \mathcal{L}=M_{P}^{2} F\left({ }^{s} R\right),{ }^{i n t} \mathcal{L}=-\sqrt{|\Lambda|}{ }^{m} \mathbf{T}_{\mu \nu} \mathbf{u}^{\mu} \mathbf{u}^{\nu} / u,{ }^{\chi} \mathcal{L}=M_{P}^{2}|\Lambda|\left(\chi^{3 / 2}+|\Lambda||u|^{2 z}\right) .
$$

In these formulas, $M_{P}$ is the Plank gravitational mass; $z=1$ if we search for compatibility with the covariant entropic gravity model elaborated in [30], or $z=2$ if we search for a limit to the standard de Sitter space solution [31, 32] criticizing certain constructions in the previous works 3 The energy-momentum tensors considered in above formulas and/or derived from respective Lagrangians in (6) are computed using variations on $\mathbf{g}^{\mu \nu}$, for instance, when

$$
{ }^{m} \mathbf{T}_{\mu \nu}:=-\frac{2}{\sqrt{|\mathbf{g}|}} \frac{\delta\left(\sqrt{|\mathbf{g}|}{ }^{m} \mathcal{L}\right)}{\delta \mathbf{g}^{\mu \nu}}=2 \frac{\delta^{m} \mathcal{L}}{\delta \mathbf{g}^{\mu \nu}}+\mathbf{g}_{\mu \nu}{ }^{m} \mathcal{L} .
$$

For the full system, the effective energy-momentum tensor is computed for

$$
{ }^{F} \mathbf{T}_{\beta \gamma}=\left[\frac{1}{2}\left(F-\frac{\partial F}{\partial^{s} R}\right) \mathbf{g}_{\beta \gamma}-\left(\mathbf{g}_{\beta \gamma} \mathbf{D}_{\alpha} \mathbf{D}^{\alpha}-\mathbf{D}_{\beta} \mathbf{D}_{\gamma}\right) \frac{\partial F}{\partial{ }^{s} R}\right]\left(\frac{\partial F}{\partial{ }^{s} R}\right)^{-1}
$$

when

$$
{ }^{t o t} \mathbf{T}_{\mu \nu}=\left(\frac{\partial F}{\partial{ }^{s} R}\right)^{-1}{ }^{m} \mathbf{T}_{\mu \nu}+{ }^{F} \mathbf{T}_{\mu \nu}+{ }^{i n t} \mathbf{T}_{\mu \nu}+{ }^{\chi} \mathbf{T}_{\mu \nu}
$$

\footnotetext{
${ }^{3}$ To elaborate a covariant version of entropic elastic gravity with a displacement-vector $\mathbf{u}$ (called also imposter field) it is supposed that the Lagrangian of the free imposter filed is of the form $\mathcal{L} \equiv(\chi)^{3 / 2}$, where $\chi$ is the kinetic term constructing from $\mathbf{u}$ as a combination for a gauged vector field, see details and motivations in 30. In our approach with geometric flow evolution, an unconventional power $3 / 2$ prescribes a subclass of models with relativistic flows of gravitational and matter fields. This allow us to conclude that entropic/ elastic gravity theories of this type can be modelled as some examples (with specific constants in effective Lagrangians) of a more general theory of nonholonomic relativistic Ricci flows.
} 
Choosing $F\left({ }^{s} R\right)={ }^{s} R$ and the Levi-Civita connection $\mathbf{D}=\nabla$, we obtain respective formulas for ${ }^{i n t} \mathbf{T}_{\mu \nu}$ and $\chi_{\mathbf{T}_{\mu \nu}}$ being similar to (10)-(13) in [31, 4 In result, the generalized source splits into three components,

$$
{ }^{t o t} \boldsymbol{\Upsilon}_{\mu \nu}:=\varkappa\left({ }^{t o t} \mathbf{T}_{\mu \nu}-\frac{1}{2} \mathbf{g}_{\mu \nu}{ }^{t o t} \mathbf{T}\right)=\left(\frac{\partial F}{\partial^{s} R}\right)^{-1}{ }^{m} \boldsymbol{\Upsilon}_{\mu \nu}+{ }^{F} \boldsymbol{\Upsilon}_{\mu \nu}+{ }^{i n t} \boldsymbol{\Upsilon}_{\mu \nu}+{ }^{\chi} \mathbf{\Upsilon}_{\mu \nu}
$$

where $\varkappa$ is determined in standard form by the Newton gravitational constant $G$.

Let us consider effective sources which via $\mathrm{N}$-adapted frames can be parameterized in the form

$$
\boldsymbol{\Upsilon}_{\nu}^{\mu}(\tau)=\mathbf{e}^{\mu^{\prime}}(\tau) \mathbf{e}_{\nu}{ }^{\nu^{\prime}}(\tau)\left[{ }^{t o t} \boldsymbol{\Upsilon}_{\mu^{\prime} \nu^{\prime}}(\tau)-\frac{1}{2} \partial_{\tau} \mathbf{g}_{\mu^{\prime} \nu^{\prime}}(\tau)\right]=\left[{ }_{h} \Upsilon\left(\tau, x^{k}\right) \delta_{j}^{i}, \Upsilon\left(\tau, x^{k}, y^{3}\right) \delta_{b}^{a}\right]
$$

for families of vielbein transforms $\mathbf{e}_{\mu^{\prime}}^{\mu}(\tau)=\mathbf{e}_{\mu^{\prime}}^{\mu}\left(\tau, u^{\gamma}\right)$ and their duals $\mathbf{e}_{\nu}{ }^{\nu^{\prime}}\left(\tau, u^{\gamma}\right)$, when $\mathbf{e}^{\mu}=\mathbf{e}_{\mu^{\prime}} d u^{\mu^{\prime}}$. The values $\left[{ }_{h} \Upsilon\left(\tau, x^{k}\right), \Upsilon\left(\tau, x^{k}, y^{3}\right)\right]$ can be fixed as generating functions for (effective) matter sources imposing nonholonomic frame constraints on stationary distributions or cosmological dynamics of (effective) matter fields. Such effective sources allow us to construct in explicit form exact stationary solutions with Killing symmetry on $\partial_{4}=\partial_{t}$ of the system of nonlinear PDEs (4) for families of metrics and N-connections parameterized for 4-d configurations in the form $\mathbf{g}(\tau)=\left[g_{i}(\tau), g_{a}(\tau), N_{i}^{a}(\tau)\right]$. The N-adapted coefficients of such geometric data do not depend on variable $y^{4}$ and can be parameterized in the form

$$
g_{i}(\tau)=e^{\psi\left(\tau, x^{j}\right)}, g_{a}(\tau)=h_{a}\left(\tau, x^{k}, y^{3}\right), N_{i}^{3}=w_{i}\left(\tau, x^{k}, y^{3}\right), \quad N_{i}^{4}=n_{i}\left(\tau, x^{k}, y^{3}\right) .
$$

It should be noted here that an effective source ${ }^{t o t} \boldsymbol{\Upsilon}_{\mu \nu}$ (7) and respective $\tau$-families $\boldsymbol{\Upsilon}_{\nu}^{\mu}(\tau)$ (8) do not satisfy any standard for GR energy conditions even they may include contributions from a standard energy-momentum tensor ${ }^{m} \mathbf{T}_{\mu \nu}$ for matter fields subjected to certain physically motivated strong, null, or weak energy conditions. This is typical for MGTs and in the case of nonholonomic deformations of geometric structures like in GR and nonholonomic mechanics with Lagrange multiples, when standard dynamical conservation laws can not be formulated. Re-defining the nonholonomic variables, for instance, for configurations with Levi-Civita connections, it is possible to model geometric evolution of nonholonomic systems with constraints when certain strong / null / weak conditions are preserved for well defined conditions on stationary systems. Strong energy conditions are violated for various classed of solutions in modern inflationary and accelerating cosmology (for instance, for modelling dark matter and dark energy interactions). In a more general context, for nonholonomic dynamical systems (with gravitational and effective matter field interactions) and gradient models of their geometric flow evolution, there are hierarchies of nonlinear conservation laws, see detailed discussions and references in [7, 43, 45, 9, 47.

We conclude that an analogous emergent gravity in E. Verlinde sense [3, 24, 30], can be constructed for Lagrange distributions (6) and respective sources (77) introduced as generating data for the nonholonomic Hamilton equations (44) and respective relativistic Ricci solitons. Such geometric flow evolution theories and their spacetime "elastic" properties are determined by the generalized $\mathcal{W}$-entropy (3).

\section{Geometric flow evolution to stationary quasi-periodic structures}

Following the geometric procedure for the AFDM described in Tables 1 and 2 and appendix A in [43, see also details in [33, 34, 35, we can decouple and integrate with stationary configurations the generalized Hamilton equations (44). In this section, we explain in brief how such quasi-stationary (not depending on the time like variable in corresponding $\mathrm{N}$-adapted coordinates) and quasi-periodic solutions can be generated by relativistic geometric flows. Corresponding quadratic linear forms are determined by distinguished metrics,

\footnotetext{
${ }^{4}$ We use a system of notation which similar to [43, 5, 6, 7, 8, but different from [30, 31, 32.
} 
d-metrics, (with the coefficients written with respect to a $\mathrm{N}$-adapted base) can be parameterized in this form:

$$
\begin{aligned}
d s^{2}= & e^{\psi(\tau)}\left[\left(d x^{1}\right)^{2}+\left(d x^{2}\right)^{2}\right]-\frac{\left(\partial_{3} h_{4}(\tau)\right)^{2}}{\left|\int d y^{3} \Upsilon(\tau)\left(\partial_{3} h_{4}(\tau)\right)\right| h_{4}(\tau)}\left[d y^{3}+\frac{\left.\partial_{i}\left(\int d y^{3} \Upsilon(\tau) \partial_{3} h_{4}(\tau)\right]\right)}{\Upsilon(\tau) \partial_{3} h_{4}(\tau)} d x^{i}\right]^{2} \\
& +h_{4}(\tau)\left[d t+\left({ }_{1} n_{k}(\tau)+4{ }_{2} n_{k}(\tau) \int d y^{3} \frac{\left(\partial_{3} h_{4}(\tau)\right)^{2}}{\left|\int d y^{3} \Upsilon(\tau)\left(\partial_{3} h_{4}(\tau)\right)\right|\left(h_{4}(\tau)\right)^{5 / 2}}\right) d x^{k}\right]^{2},
\end{aligned}
$$

where ${ }_{1} n_{k}(\tau)={ }_{1} n_{k}\left(\tau, x^{i}\right)$ and ${ }_{2} n_{k}(\tau)={ }_{2} n_{k}\left(\tau, x^{i}\right)$ are integration functions, $h_{4}(\tau)=h_{4}\left(\tau, x^{i}\right)$ can be taken as a generating function, and $\psi(\tau)=\psi(\tau, r, \theta)$ is a solution of a 2-d Poisson equation (see below the definitions and formulas (20), (13) and (12)), $\partial_{11}^{2} \psi+\partial_{22}^{2} \psi=2{ }_{h} \Upsilon$. Here we note that integration functions/ constants appear as general ones when certain integrals are computed and such values are not present in the fundamental field/ evolution equations. We need, for instance, to consider certain boundary/ initial conditions in order to define explicit values for an integration function/ constant. Generating functions are present in direct or indirect forms in fundamental field/ evolution equations and their type and (non) linear symmetries determine explicitly the class of constructed solutions and their basic physical properties. Such solutions are, in general, with nontrivial nonholonomically induced torsion which allows us to extract LC-configurations by imposing additional constraints, see footnote 8 ,

\subsection{Stationary quasicrystal spacetime and effective matter configurations}

To elaborate toy models of dark matter and dark energy theories certain quasi-periodic, filament, nonlinear wave etc. structures were studied in [43, 45], see also references therein 5 For simplicity, in this work, we consider simplified quasi-periodic models defined by quasicrystal, QC, structures and analogous dynamic phase field crystal models which can be generated using a generic flow evolution on parameter $\tau$. A QC structure can be defined by generating functions $\bar{q}=\bar{q}\left(x^{i}, y^{3}, \tau\right)$ defined as a solution of an evolution equation with conserved dynamics,

$$
\frac{\partial \bar{q}}{\partial \tau}={ }^{b} \widehat{\Delta}\left[\frac{\delta \bar{F}}{\delta \bar{q}}\right]=-{ }^{b} \widehat{\Delta}\left(\Theta \bar{q}+Q \bar{q}^{2}-\bar{q}^{3}\right)
$$

Such an evolution is considered on a 3-d spacelike hypersurface $\Xi_{t}$ when the canonically nonholonomically deformed hypersurface Laplace operator ${ }^{b} \widehat{\Delta}:=\left({ }^{b} \widehat{D}\right)^{2}=b^{i} \grave{D_{i}} \widehat{D}_{\grave{j}}$, where indices span the values of $i, j, \ldots 1,2,3$. This operator is a distortion of ${ }^{b} \Delta:=\left({ }^{b} \nabla\right)^{2}$ constructed in 3-d Riemannian geometry. The functional $\bar{F}$ in (11) is an effective free energy

$$
\bar{F}[\bar{q}]=\int\left[-\frac{1}{2} \bar{q} \Theta \bar{q}-\frac{Q}{3} \bar{q}^{3}+\frac{1}{4} \bar{q}^{4}\right] \sqrt{b} d x^{1} d x^{2} \delta y^{3},
$$

where $b=\operatorname{det}\left|b_{i j}\right|, \delta y^{3}=\mathbf{e}^{3}$ and the operators $\Theta$ and $Q$ are explained in [43], see also Appendix to this paper. This class of nonlinear interactions is stabilized by the cubic term and the second order resonant

\footnotetext{
${ }^{5}$ As a quasi-periodic crystal, or quasicrystal (QC), we consider a structure that is ordered but not periodic (as it used in modern literature). It is defined by quasi-periodic pattern which can continuously fill all available space, it lacks translational symmetry on a space coordinate, or all space coordinates; and, for time (quasi) crystals, on a time like coordinate. In this work, the translational symmetry on geometric evolution parameter $\tau$ can be also broken. Here we note that toy models with (locally) anisotropic geometric evolution can be similarly elaborated with aperiodic space and/or evolution order, which is not the same as quasi-periodic configurations. For other type models, we can consider one-dimensional Fibonacci chains, two-dimensional Penrose tiles; the four dimensional Elser-Sloane QC derived from the cut-and-project method of $E-8$ lattice in eight dimensions and other examples of QCs. Of course, observable anisotropic non-homogeneous matter filaments in modern cosmology are not necessarily described as QCs. Such realistic structure can be modelled as nonholonomic deformations under geometric and time like evolution of some quasi-periodic or aperiodic configurations. This requests a more advanced and cumbersome geometric and numeric techniques.
} 
interactions are varied by setting an observable value of $Q$, for instance, for certain quasi-periodicity of cosmological structure. The average value $\langle\bar{q}\rangle$ is conserved for any fixed $t$ and/or $\tau_{0}$. Fixing a constant $\tau_{0}$, we generate quasi-periodic Ricci solitons determined by an effective parameter $\bar{q}$ of the system. We can choose $\left\langle\bar{q}>_{\mid \tau=\tau_{0}}=0\right.$ when other values are accommodated by redefining the normalization function $f$ and operator $\Theta$ and $Q$.

Off-diagonal stationary metrics of type (10) define two classes of exact solutions of (4) if

$$
\begin{array}{ccc}
h_{4}(\tau) & = & \bar{q}\left(\tau, x^{i}, y^{3}\right), \text { for gravitational QC configurations ; } \\
\Upsilon(\tau) & = & \Upsilon[\bar{q}(\tau)]=\Upsilon\left[\bar{q}\left(\tau, x^{i}, y^{3}\right)\right], \text { for elastic QC structures for DM. }
\end{array}
$$

Fixing a QC source for $\Upsilon(\tau)$, parameterized in N-adapted form (8), as a functional of a solution (11), we prescribe a quasi-periodic evolution/dynamics for effective fields $\chi$ and/or $\mathbf{u}$ in (6). This imposes QC sources ${ }^{\text {int }} \boldsymbol{\Upsilon}_{\mu \nu}$ and/or ${ }^{\chi} \boldsymbol{\Upsilon}_{\mu \nu}$ in (7). Details on generating such pattern forming, space QC and/or time and space QC structures with applications in modern cosmology can be found in [43, 45, 33, 34, 35]. Relativistic evolution scenarios, with suppersymmetric or noncommutative variables, Finsler like generalizations are studied in [5, 6, 7, 8] and references therein. In those works, there were used different types of effective Lagrangians and nonholonomic Perelman functionals with possible dependence on time like coordinates.

Prescribing respective data for geometric evolution of stationary Ricci soliton models, we impose certain nonholonomic frame constraints on geometric evolution and self-similar configurations of stationary d-metrics. In such cases, we write

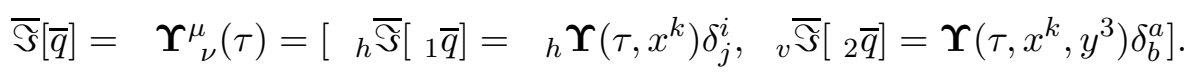

"Overlined" symbols are used in order to emphasize that such (effective) are determined by quasi-periodic data of type (12) (which can be different for horizontal configurations, ${ }_{1} \bar{q}$, and vertical configurations ${ }_{2} \bar{q}$ ) considered for geometric flow and/or Ricci soliton systems on nonlinear PDEs.

\subsection{Nonlinear PDEs for the geometric flow evolution of stationary quasi-periodic con- figurations}

In canonical nonholonomic variables with functional dependence of d-metrics and effective sources on some prescribed classes of QC structures, the system of nonholonomic relativistic flow equations (4) can be written in the form (5) but with geometric objects depending additionally on a temperature like parameter $\tau$ and for effective source (13),

$$
\mathbf{R}_{\alpha \beta}[\bar{q}]=\bar{\Im}_{\alpha \beta}[\bar{q}] .
$$

It should be noted that such PDEs are obtained for an undetermined normalization function $f(\tau)=f\left(\tau, u^{\gamma}\right)$. Such a function can be chosen in a form which allows us to construct exact/parametric solutions. The constructions can be redefined in other systems of reference and with re-defined evolution parameters chosen for an explicit modeling of certain evolution/ dynamical scenarios for quasi-periodic configurations. For self-similar point $\tau=\tau_{0}$ configurations with $\partial_{\tau} \mathbf{g}_{\mu \nu}\left(\tau_{0}\right)=0$, this system of nonlinear PDEs transforms into the canonical nonholonomic Ricci soliton equations (5).

In this work, we study stationary quasi-periodic configurations which can be described (for respective systems of reference/ coordinates) by coefficients of the d-metrics and derived geometric objects do not depend on $y^{4}=t$ with respect to a class of $\mathrm{N}$-adapted frames. Such solitons possess a Killing symmetry on $\partial_{4}$. Using a source $\bar{\Im}[\bar{q}]=\left[{ }_{h} \bar{\Im}\left[{ }_{1} \bar{q}\right],{ }_{v} \bar{\Im}\left[{ }_{2} \bar{q}\right]\right]$ (13), we compute the nontrivial N-adapted coefficients of the Ricci d-tensor. The end result of the geometric flow modified Einstein equations (14) is that they can be 
written in the form

$$
\begin{aligned}
& \left.\left.\mathbf{R}_{1}^{1}{ }_{1} \bar{q}\right]=\mathbf{R}_{2}^{2}{ }_{1} \bar{q}\right]=-{ }_{h} \bar{\Im}\left[{ }_{1} \bar{q}\right] \text { i.e. } \frac{g_{1}^{\bullet} g_{2}^{\bullet}}{2 g_{1}}+\frac{\left(g_{2}^{\bullet}\right)^{2}}{2 g_{2}}-g_{2}^{\bullet \bullet}+\frac{g_{1}^{\prime} g_{2}^{\prime}}{2 g_{2}}+\frac{\left(g_{1}^{\prime}\right)^{2}}{2 g_{1}}-g_{1}^{\prime \prime}=-2 g_{1} g_{2} \bar{S} \bar{\Im} ; \\
& \mathbf{R}_{3}^{3}\left[{ }_{2} \bar{q}\right]=\mathbf{R}_{4}^{4}\left[{ }_{2} \bar{q}\right]=-{ }_{v} \bar{\Im}\left[{ }_{2} \bar{q}\right] \text { i.e. } \frac{\left(h_{4}^{\diamond}\right)^{2}}{2 h_{4}}+\frac{h_{3}^{\diamond} h_{4}^{\diamond}}{2 h_{3}}-h_{4}^{\diamond}=-2 h_{3} h_{4} \quad{ }_{v} \bar{\Im} ; \\
& \mathbf{R}_{3 k}(\tau)=-w_{k}\left[\left(\frac{h_{4}^{\diamond}}{2 h_{4}}\right)^{2}+\frac{h_{3}^{\diamond}}{2 h_{3}} \frac{h_{4}^{\diamond}}{2 h_{4}}-\frac{h_{4}^{\diamond}}{2 h_{4}}\right]+\frac{h_{4}^{\diamond}}{2 h_{4}}\left(\frac{\partial_{k} h_{3}}{2 h_{3}}+\frac{\partial_{k} h_{4}}{2 h_{4}}\right)-\frac{\partial_{k} h_{4}^{\diamond}}{2 h_{4}}=0, \\
& \mathbf{R}_{4 k}(\tau)=\frac{h_{4}}{2 h_{4}} n_{k}^{\diamond}+\left(\frac{3}{2} h_{4}^{\diamond}-\frac{h_{4}}{h_{3}} h_{3}^{\diamond}\right) \frac{n_{k}^{\diamond}}{2 h_{3}}=0,
\end{aligned}
$$

where, for instance, $h_{3}^{\diamond}=\partial_{3} h_{3} \cdot 6$

Further simplifications of the system of nonlinear PDEs describing the nonholonomic geometric evolution are possible if we consider certain classes of nonlinear symmetries of such systems. Let us introducing the coefficients $\alpha_{i}=\left(\partial_{3} h_{4}\right)\left(\partial_{i} \varpi\right), \beta=\left(\partial_{3} h_{4}\right)\left(\partial_{3} \varpi\right), \gamma=\partial_{3}\left(\ln \left|h_{4}\right|^{3 / 2} /\left|h_{3}\right|\right)$, where

$$
\varpi=\ln \left|\partial_{3} h_{4} / \sqrt{\left|h_{3} h_{4}\right|}\right|
$$

are considered for nonsingular values of $\partial_{3} h_{a} \neq 0$ and $\partial_{t} \varpi \neq 0$. The end-result is that we can express (15)(18), respectively, in the form

$$
\psi^{\bullet \bullet}+\psi^{\prime \prime}=2{ }_{h} \bar{\Im}\left[{ }_{1} \bar{q}\right], \quad \varpi^{\diamond} h_{4}^{\diamond}=2 h_{3} h_{4}{ }_{v} \bar{\Im}\left[{ }_{2} \bar{q}\right], \beta w_{i}-\alpha_{i}=0, \quad n_{k}^{\diamond}+\gamma n_{k}^{\diamond}=0 .
$$

Such a system can be integrated in an explicit general and/or parametric form (see details in [5, 6, 7, 8, 43, 45]) if there are prescribed a generating function $\Psi(\tau)=\Psi\left(\tau, x^{i}, y^{3}\right)=\Psi[\bar{q}]:=e^{\varpi}$, with a different QC structure, and generating sources $h \widehat{\Im}$ and ${ }_{v} \widehat{\Im}$ with another types of QC structures 7 . The functions appearing in equations (20) are the ones to be employed in d-metric (10).

We have to solve a nonlinear system of two equations for $\varpi$ in (19) and (20) involving four functions $\left(h_{3}, h_{4},{ }_{v} \bar{\Im}\left[{ }_{2} \bar{q}\right]\right.$, and $\left.\Psi\right)$. By straightforward computations using other type functionals and/or effective sources, we can check that there an important nonlinear symmetry. It allows to redefine the generating functions and effective sources (in particular, to introduce a family of effective cosmological constants ${ }_{h} \Lambda(\tau)$ and ${ }_{v} \Lambda(\tau)=\Lambda(\tau)$ with $\Lambda(\tau) \neq 0, \Lambda\left(\tau_{0}\right)=$ const, not depending on spacetime coordinates $\left.u^{\alpha}\right)$. The nonlinear symmetries are described by nonlinear transforms $\left(\Psi(\tau),{ }_{v} \bar{\Im}(\tau)\right) \Longleftrightarrow(\Phi(\tau), \Lambda(\tau))$ subjected to formulas

$$
\Lambda\left(\Psi^{2}\left[{ }_{1} \bar{q}\right]\right)^{\diamond}=\left|{ }_{v} \bar{\Im}\left[{ }_{2} \bar{q}\right]\right|\left(\Phi^{2}[\bar{q}]\right)^{\diamond} \text {, or } \Lambda \Psi^{2}\left[{ }_{1} \bar{q}\right]=\Phi^{2}[\bar{q}]\left|{ }_{v} \bar{\Im}\left[{ }_{2} \bar{q}\right]\right|-\int d y^{3} \Phi^{2}[\bar{q}]\left|{ }_{v} \bar{\Im}\left[{ }_{2} \bar{q}\right]\right|^{\diamond}
$$

\footnotetext{
${ }^{6}$ This system of nonlinear PDEs with quasi-periodic sources (15)- (18) has a very important decoupling property: Using the equation (15), we can find the coefficient $g_{1}$ (or, inversely, the coefficient $g_{2}$ ) for any prescribed functional of a stationary quasiperiodic structure encoded into a h-source ${ }_{h} \bar{\Im}\left[{ }_{1} \bar{q}\right]$ and any given coefficient $g_{2}\left(\tau, x^{i}\right)=g_{2}\left[{ }_{1} \bar{q}\right]\left(\right.$ or, inversely, $\left.g_{1}\left(\tau, x^{i}\right)=g_{1}\left[{ }_{1} \bar{q}\right]\right)$. It should be noted that the QC structure for the coefficients of a h-metric can be different from the data for and effective h-source. At the next step, we can integrate on $y^{3}$ in equation (16) and define $h_{3}\left(\tau, x^{i}, y^{3}\right)$ as a solution of first order PDE for any prescribed v-source $v_{v} \widetilde{\Im}\left[{ }_{2} \bar{q}\right]$ and given coefficient $h_{4}\left(\tau, x^{i}, y^{3}\right)=h_{4}\left[{ }_{2} \bar{q}\right]$. We can follow an inverse procedure and define $h_{4}\left(\tau, x^{i}, y^{3}\right)$ if $h_{3}\left(\tau, x^{i}, y^{3}\right)=h_{3}\left[{ }_{2} \bar{q}\right]$. The coefficients of v-metrics involve, in general, different types of QC structures comparing to those prescribed for the effective v-source. If the values of $h_{3}$ and $h_{4}$ are defined as certain QC configurations, the equations (17) transform into a system of algebraic linear equations for $w_{k}\left(\tau, x^{i}, y^{3}\right)=w_{k}[\iota]$ which, in general, are with a different QC structure. Integrating two times on $y^{3}$ in equation (18), we can compute $n_{k}\left(\tau, x^{i}, y^{3}\right)=n_{k}[\bar{q}]$ for any defined $h_{3}$ and $h_{4}$. The QC structures encoded in the coefficients of a N-connection are different (in general) from the QC encoded in the coefficients of a d-metric and nontrivial effective sources used for constructing a solution of nonholonomic geometric flow or Ricci soliton equations. Finally, we emphasize that the decoupling of such systems of nonlinear PDEs is one of the main achievements of the AFDM.

${ }^{7}$ we can construct nontrivial non-stationary solutions if such conditions are not satisfied, see examples in just cited works
} 
This way we can introduce a new generating function $\Phi\left(\tau, x^{i}, y^{3}\right)=\Phi[\bar{q}]$ which can be more convenient for integrating PDEs and expressing in a simplified form different classes of solutions. The flow running values $\Lambda(\tau)$ can be chosen from certain physical considerations when the geometric/physical data for $\Phi$ encode nonlinear symmetries and QC configurations for ${ }_{v} \bar{\Im}$, and $\Psi$. Solutions with $\Lambda=0$ have to be studied by applying more special methods, see details and examples in [43, 45]. Here we note that using nonlinear symmetries (21), we can describe the PDEs and their solutions by two equivalent sets of generating data $(\Psi, \Upsilon)$ or $(\Phi, \Lambda) 8$

\section{Computing Perelman thermodynamic variables instead of Bekenstein- Hawking ones}

Generic off-diagonal stationary solutions with QC structure for the geometric flow evolution and/or Ricci soliton, or (modified) Einstein equations of type (4) are not subjected, in general, to any surface-area, horizon, or duality conditions for describing such configurations. The thermodynamic properties of spacetimes under geometric evolution on $\tau$, and/or for a fixed $\tau_{0}$ for relativistic dynamic gravitational and (effective) matter field equations, cannot be characterized by the Bekenstein-Hawking entropy and temperature. The Perelman entropy can be defined even in the absence of horizons and when there are not conformal boundaries, nor cosmological horizons, nor Noether charges on the horizons, nor asymptotic charges at conformal infinity, etc.

We introduce a $3+1$ decomposition with local coordinates $u^{i^{\prime}}=x^{i^{\prime}}=\left(x^{1}, x^{2}, x^{3}\right)$ and $u^{4^{\prime}}=t$, which is additional to the $2+2$ decomposition, when for a (10) $\mathbf{g}=\left[g_{i j}, g_{a b}, N_{i}^{a}\right]=\left\{b_{i^{\prime} j^{\prime}}=\left(b_{i j}, b_{3}\right), \breve{N}\right\}$, where

$$
b_{i^{\prime} j^{\prime}}=\operatorname{diag}\left(b_{i^{\prime}}\right)=\left[b_{i}=g_{i}\left(\tau, x^{k}\right), b_{3}=h_{3}=-\frac{\left(\partial_{3} h_{4}\left(\tau, x^{k}, x^{3}\right)\right)^{2}}{\left|\int d y^{3} \Upsilon\left(\tau, x^{k}, x^{3}\right)\left(\partial_{3} h_{4}\left(\tau, x^{k}, x^{3}\right)\right)\right| h_{4}\left(\tau, x^{k}, x^{3}\right)}\right]
$$

on a hypersurface $\Xi_{t}$ and $\breve{N}^{2}=-h_{4}\left(\tau, x^{k}, x^{3}\right)$ is the lapse function. The N-connection coefficients are parameterized $N_{i}^{3}=w_{i}\left(\tau, x^{k}, x^{3}\right)$ and $N_{i}^{4}=n_{i}\left(\tau, x^{k}, x^{3}\right)$. For such new classes of stationary and cosmological solutions in MGTs and GR, we suggested [6, 7, 8] to elaborate on statistical and geometric thermodynamics models using the corresponding generalizations of the Perelman F- and W-entropy.

\subsection{Geometric thermodynamics for relativistic nonholonomic Ricci flows}

For stationary configurations, we can characterise the geometric flows by analogous thermodynamic systems on corresponding families of 3-d closed hypersurfaces $\Xi_{t}$ using $, \mathcal{W}:=\mathcal{W}(\tau)_{\mid \Xi_{t}}$ which is constructed using respective 3 -d projections with data $\left(b_{i j}, b_{3}\right)$ and $, \mathbf{D}:=\mathbf{D}_{\mid \Xi_{t}}$ nonholonomically deformed W-entropy , $\mathcal{W}$, see details in section 5.1 of [6] and [33, 34, 35]. In such an approach (in this paper omitting hats on geometric values), it is considered a standard partition function

$$
\mathcal{Z}[\mathbf{g}(\tau)]=\int_{t_{1}}^{t_{2}} \int_{\Xi_{t}}(4 \pi \tau)^{-2} e^{-f} \sqrt{|\mathbf{g}|} d^{4} u(-f+2), \text { for } \mathbf{V} .
$$

for the conditions stated for definitions (2) and (3) and when the scaling function $f$ satisfies the normalization formulas $\int_{t_{1}}^{t_{2}} \int_{\Xi_{t}} M \sqrt{\left|\mathbf{g}_{\alpha \beta}\right|} d^{4} u=1$ for $M=(4 \pi \tau)^{-3} e^{-f}$. In principle, we can fix any convenient normalization which allows us to solve certain important systems of nonlinear PDEs, to compute the statistical/geometric thermodynamic variables, and then to recompute and re-scale the geometric flow scenario in a covariant form. Then we can and elaborate on physical models prescribing certain generation and integration data which are

\footnotetext{
${ }^{8}$ Such nonlinear symmetries can be considered in GR, when the zero torsion conditions, equivalently, Levi-Civita, LCconditions for stationary configurations (9) can be extracted if it is solved additionally a system of 1st order PDEs, $\partial_{3} w_{i}=\left(\partial_{i}-w_{i} \partial_{3}\right) \ln \sqrt{\left|h_{3}\right|},\left(\partial_{i}-w_{i} \partial_{3}\right) \ln \sqrt{\left|h_{4}\right|}=0, \partial_{k} w_{i}=\partial_{i} w_{k}, \partial_{3} n_{i}=0, \partial_{i} n_{k}=\partial_{k} n_{i}$.
} 
compatible with experimental/observational date and provide certain predictibility for nonlinear evolution processes. This allows us to compute for d-metrics (10) all necessary thermodynamical variables as it was constructed in [4, 39] for the dimension $n=3$ and then to extend to 4-d relativistic configurations. This way, a statistical model can be elaborated for any $\mathcal{Z}$ associated to a $Z=\int \exp (-\beta E) d \omega(E)$ for a canonical ensemble at temperature $\beta^{-1}=\tau$ and measure taken for a density of states $\omega(E)$. Using such constructions, the standard thermodynamic variables are computed for the average energy, $\mathcal{E}=\langle E\rangle:=-\partial \log Z / \partial \beta$, the

entropy $S:=\beta\langle E\rangle+\log Z$ and the fluctuation $\sigma:=\left\langle(E-\langle E\rangle)^{2}\right\rangle=\partial^{2} \log Z / \partial \beta^{2}$. Here we note that for nonrelativisitc configurations there is a key analogy between the generalized Ricci flow equations (44) with the heat diffusion equation.

Using (23) and (3) and respective $3+1$ parameterizations of d-metrics (22) with a time-like coordinate $y^{4}=t$ and temperature-like evolution parameter $\tau$, we define and compute analogous thermodynamic variables for geometric evolution flows of stationary gravitational configurations,

$$
\begin{aligned}
\mathcal{E} & =-\tau^{2} \int_{t_{1}}^{t_{2}} \int_{\Xi_{t}}(4 \pi \tau)^{-2} e^{-f} \sqrt{\left|q_{1} q_{2} \mathbf{q}_{3}\left({ }_{q} N\right)\right|} \delta^{4} u\left({ }_{s} R+|\mathbf{D} f|^{2}-\frac{2}{\tau}\right), \\
\mathcal{S} & =-\int_{t_{1}}^{t_{2}} \int_{\Xi_{t}}(4 \pi \tau)^{-2} e^{-f} \sqrt{\left|q_{1} q_{2} \mathbf{q}_{3}\left({ }_{q} N\right)\right|} \delta^{4} u\left[\tau\left({ }_{s} R+|\mathbf{D} f|^{2}\right)+f-4\right], \\
\eta & =-2 \tau^{4} \int_{t_{1}}^{t_{2}} \int_{\Xi_{t}}(4 \pi \tau)^{-2} e^{-f} \sqrt{\left|q_{1} q_{2} \mathbf{q}_{3}\left({ }_{q} N\right)\right|} \delta^{4} u\left[\left|\mathbf{R}_{\alpha \beta}+\mathbf{D}_{\alpha} \mathbf{D}_{\beta} f-\frac{1}{2 \tau} \mathbf{g}_{\alpha \beta}\right|^{2}\right],
\end{aligned}
$$

where $\delta^{4} u=\mathbf{e}^{\alpha}$ contains N-elongated differentials (A.3) in order to compute such integrals in N-adapted forms.

These formulas are related by distortion formulas with corresponding values determined by the LeviCivita connection $\nabla$, see details and results of such computations for metrics of type (10) and various quasi-periodic, cosmological, black hole deformed structures etc. in [9, 33, 34, 35, 6, 17, 8].

\subsection{Computing Perelman's thermodynamic variables for stationary quasi-periodic ge- ometric flows}

We show how G. Perelman's W-entropy and related thermodynamic variables can be computed for stationary quasi-periodic systems and nonholonomically deformed black hole, BH, solutions under geometric flow evolution, see details and references in [46, 7, 43, 34. Such BH solutions can be with a deformed horizon, for instance, of ellipsoid type but different from the Kerr one because of presence of certain generic offdiagonal terms of metrics. More general classes of such stationary solutions describe BHs with spherical and ellipsoid horizons imbedded in locally anisotropic backgrounds (defined by solitonic waves or quasi-periodic structures), with polarization of physical constants etc. Even though for some special ellipsoid configurations it is possible to define analogs of hypersurface Bekenstein-Hawking entropy, a complete thermodynamic description of such locally anisotropic gravitational and matter field systems is not possible using the standard $\mathrm{BH}$ thermodynamics paradigm. In our works, we show that using relativistic nonholonomic extensions of the G. Perelman geometric flow thermodynamics, such a description is possible for all types of exact and approximate solutions in (modified) gravity theories because all values / thermodynamic variables like (23), (3) and (24) can be computed at least along a set of causal curves covering a spacetime region if a d-metric $\mathrm{g}$ and respective canonical d-connection, or associated LC-connection, are found as solutions for certain important systems of nonlinear PDEs (of geometric flow evolution, or dynamical ones).

\subsubsection{Fixing normalization and integration functions and effective cosmological constants}

To analyze main properties geometric evolution of (14) when $\mathbf{R}_{\alpha \beta}=\bar{\Im}_{\alpha \beta}$ and ${ }_{s} R=\bar{\Im}_{a}^{a}$, we can chose a constant value for the normalizing function, $f(\tau)=f_{0}=$ const $=0$. This prescribes a geometric vertical 
scale for flow evolution determined by data $(\Phi(\tau), \Lambda(\tau))$ of such physical models and related via nonlinear symmetries (21) to a generating source ${ }_{v} \bar{\Im}(\tau)$ (such a h-scale is determined by a 2-d Poisson equation as described by fist formula in (20) $)$. We can consider additionally certain new constants for integration functions, which simplify substantially the formulas for G. Perelman's thermodynamic variables 9 Here we note that if arbitrary integration functions are considered, we generate solutions for the canonical d-connection with non-trivial nonholonomic induced torsion. Zero torsion conditions for the Levi-Civita configurations can be satisfied for a subclass of integration functions, or imposing additional constraints on such integration functions depending on some spacetime coordinates and, in general, on a geometric flow parameter. The formulas for the F- and $\mathrm{W}$-functionals (2) and (3) for d-metrics (10) with parameterizations are written

$$
\begin{aligned}
& \overline{\mathcal{F}}=\frac{1}{8 \pi^{2}} \int \tau^{-2} \sqrt{|\mathbf{g}[\bar{\Phi}(\tau)]|} \delta^{4} u\left[{ }_{h} \Lambda(\tau)+\Lambda(\tau)\right], \text { and } \\
& \overline{\mathcal{W}}=\frac{1}{4 \pi^{2}} \int \tau^{-2} \sqrt{|\mathbf{g}[\bar{\Phi}(\tau)]|} \delta^{4} u\left(\tau\left[{ }_{h} \Lambda(\tau)+\Lambda(\tau)\right]^{2}-1\right),
\end{aligned}
$$

where $\sqrt{|\overline{\mathbf{g}}(\tau)|}=\sqrt{|\mathbf{g}[\Phi(\tau)]|}=\sqrt{\left|q_{1} q_{2} \mathbf{q}_{3}\left({ }_{q} N\right)\right|}=2 e^{\psi(\tau)}|\bar{\Phi}(\tau)| \sqrt{\frac{\left|\left[\bar{\Phi}^{2}(\tau)\right]^{\triangleright}\right|}{\left|\Lambda(\tau) \int d y^{3}{ }_{v}(\tau)\left[\Phi^{2}(\tau)\right]^{\diamond}\right|}}$ is computed for d-metrics parameterized in the form (22) with $h_{4}^{[0]}=0$,

$$
q_{1}(\tau)=q_{2}(\tau)=e^{\psi(\tau)}, \mathbf{q}_{3}(\tau)=-\frac{4\left[\bar{\Phi}^{2}(\tau)\right]^{\diamond}}{\left|\int d y^{3}{ }_{v} \bar{\Im}(\tau)\left[\bar{\Phi}^{2}(\tau)\right]^{\diamond}\right|},\left[{ }_{q} N(\tau)\right]^{2}=h_{4}\left(\tau, x^{k}, y^{3}\right)=\bar{h}_{4}(\tau)=-\frac{\bar{\Phi}^{2}(\tau)}{4 \Lambda(\tau)} .
$$

We can encode QC structures using $\bar{\Phi}(\tau)=\Phi\left({ }_{4} \bar{q}\right)$ or $\bar{h}_{4}(\tau)=h_{4}\left(\tau, x^{k}, y^{3}\right)=h_{4}\left({ }_{4} \bar{q}\right)$. The N-adapted differential encode also quasi-periodic stationary configurations

$$
\delta^{4} u=d x^{1} d x^{2} \mathbf{e}^{3} \mathbf{e}^{4}=d x^{1} d x^{2}\left[d y^{3}+\bar{w}_{i}(\tau) d x^{i}\right]\left[d t+n_{i}(\tau) d x^{i}\right]
$$

because there are considered respective values of N-connection coefficients when

$$
N_{i}^{a}=\left[\bar{w}_{i}(\tau)=\frac{\partial_{i}\left(\int d y^{3}{ }_{v} \bar{\Im}(\tau)\left[\bar{\Phi}^{2}(\tau)\right]^{\diamond}\right)}{{ }_{v} \bar{\Im}(\tau)\left[\bar{\Phi}^{2}(\tau)\right]^{\diamond}}, \bar{n}_{i}(\tau)=0\right]
$$

for fixed integration functions ${ }_{1} n_{k}(\tau)=0$ and ${ }_{2} n_{k}(\tau)=0$.

The thermodynamic generating function $\overline{\mathcal{Z}}(\underline{23})$ corresponding to $\overline{\mathcal{W}}(25)$ and fixed $\widehat{f}$-normalization is

$$
\overline{\mathcal{Z}}[\mathbf{g}(\tau)]=\frac{1}{4 \pi^{2}} \int \tau^{-2} d \overline{\mathcal{V}}(\tau)
$$

where the effective integration volume functional $d \overline{\mathcal{V}}(\tau)=d \mathcal{V}\left(\psi(\tau), \bar{\Phi}(\tau),{ }_{v} \bar{\Im}(\tau), \Lambda(\tau)\right)$ is computed

$$
d \overline{\mathcal{V}}(\tau)=e^{\psi(\tau)}|\bar{\Phi}(\tau)| \sqrt{\frac{\left|\left[\bar{\Phi}^{2}(\tau)\right]^{\diamond}\right|}{\left|\Lambda(\tau) \int d y^{3}{ }_{v} \bar{\Im}(\tau)\left[\bar{\Phi}^{2}(\tau)\right]^{\diamond}\right|}} d x^{1} d x^{2}\left[d y^{3}+\frac{\partial_{i}\left(\int d y^{3}{ }_{v} \bar{\Im}(\tau)\left[\bar{\Phi}^{2}(\tau)\right]^{\diamond}\right)}{{ }_{v} \bar{\Im}(\tau)\left[\bar{\Phi}^{2}(\tau)\right]^{\diamond}} d x^{i}\right] d t
$$

Above formulas allow us to compute analogous thermodynamic variables for the geometric evolution of stationary quasi-periodic configurations,

$$
\begin{aligned}
& \overline{\mathcal{E}}(\tau)=-\frac{\tau^{2}}{4 \pi^{2}} \int\left(\left[{ }_{h} \Lambda(\tau)+\Lambda(\tau)\right]-\frac{2}{\tau}\right) \tau^{-2} d \overline{\mathcal{V}}(\tau), \text { and } \\
& \overline{\mathcal{S}}(\tau)=-\frac{1}{4 \pi^{2}} \int\left(\tau\left[{ }_{h} \Lambda(\tau)+\Lambda(\tau)\right]-2\right) \tau^{-2} d \overline{\mathcal{V}}(\tau) .
\end{aligned}
$$

\footnotetext{
${ }^{9}$ The idea is to computing such variables in a convenient system of reference/coordinates when the solutions and thermodynamic variables are described by simplified formulas. Then, we can consider general frame/coordinate transforms to any system of reference and curved (co)tangent Lorentz manifolds and other type normalisation for their geometric evolution.
} 
In this paper, we omit and do not provide computations/ applications of flow fluctuations $\bar{\eta}(24)$.

\subsubsection{Thermodynamic variables for stationary quasi-periodic generating functions \& sources}

Such variables are computed for a $3+1$ spitting (22) determined by a QC d-metric with coefficients of type (12) with possible constraints to LC-configurations, when

$$
\begin{aligned}
q_{1} & =q_{2}\left[{ }_{h} \bar{q}\right]=e^{\psi\left[{ }_{h} \bar{q}\right]}, \mathbf{q}_{3}\left[{ }_{v} \bar{q}\right]=-\frac{4\left[\left(\Phi\left[{ }_{4} \bar{q}\right]\right)^{2}\right]^{\diamond}}{\left|\int d y^{3}{ }_{v} \bar{\Im}\left[\left(\Phi\left[{ }_{4} \bar{q}\right]\right)^{2}\right]^{\diamond}\right|},\left[{ }_{q} N(\tau)\right]^{2}=h_{4}\left[{ }_{4} \bar{q}\right]=-\frac{\left(\Phi\left[{ }_{4} \bar{q}\right]\right)^{2}}{4 \Lambda(\tau)} \\
\text { and } N_{i}^{a} & =\left[\bar{w}_{i}(\tau)=\frac{\partial_{i}\left(\int d y^{3}{ }_{v} \bar{\Im}\left[\Phi^{2}\left[{ }_{4} \bar{q}\right]\right]^{\diamond}\right)}{{ }_{v} \bar{\Im}\left[\Phi^{2}\left[{ }_{4} \bar{q}\right]\right]^{\diamond}}, \bar{n}_{i}(\tau)=0\right] .
\end{aligned}
$$

Using the d-metric coefficients and prescribing QC distributions for the effective volume (27), we obtain

$$
d \mathcal{V}=e^{\psi\left[{ }_{h} \bar{q}\right]}\left|\Phi\left[{ }_{4} \bar{q}\right]\right| \sqrt{\frac{\left|\left[\Phi^{2}\left[{ }_{4} \bar{q}\right]\right]^{\diamond}\right|}{\left|\Lambda(\tau) \int d y^{3}{ }_{v} \bar{\Im}\left[\Phi^{2}\left[{ }_{4} \bar{q}\right]\right]^{\diamond}\right|}} d x^{1} d x^{2}\left[d y^{3}+\frac{\partial_{i}\left(\int d y^{3}{ }_{v} \bar{\Im}\left[\Phi^{2}\left[{ }_{4} \bar{q}\right]\right]^{\diamond}\right)}{{ }_{v} \bar{\Im}\left[\Phi^{2}\left[{ }_{4} \bar{q}\right]\right]^{\diamond}} d x^{i}\right] d t
$$

and respective thermodynamic generating function (26) $\mathcal{Z}\left[{ }_{h} \bar{q},{ }_{4} \bar{q}, \bar{q}\right]=\frac{1}{4 \pi^{2}} \int \tau^{-2} d \mathcal{V}\left[{ }_{h} \bar{q},{ }_{4} \bar{q}, \bar{q}\right]$.

The value $\mathcal{Z}\left[{ }_{h} \bar{q},{ }_{4} \bar{q}, \bar{q}\right]$ determine the thermodynamic variables (28) for geometric flows of such stationary QC structures,

$$
\begin{aligned}
\mathcal{E}\left[{ }_{h} \bar{q},{ }_{4} \bar{q}, \bar{q}\right] & =-\frac{\tau^{2}}{4 \pi^{2}} \int\left(\left[{ }_{h} \Lambda(\tau)+\Lambda(\tau)\right]-\frac{2}{\tau}\right) \tau^{-2} d \mathcal{V} \text { and } \\
\mathcal{S}\left[{ }_{h} \bar{q},{ }_{4} \bar{q}, \bar{q}\right] & =-\frac{1}{4 \pi^{2}} \int\left(\tau\left[{ }_{h} \Lambda(\tau)+\Lambda(\tau)\right]-2\right) \tau^{-2} d \mathcal{V} .
\end{aligned}
$$

For nonholonomic Ricci soliton configurations, we take such values for a fixed flow parameter $\tau_{0}$.

\subsubsection{Perelman's thermodynamics for BHs deformed by stationary quasi-periodic structures}

Such stationary systems under geometric flow evolution encode d-metrics (10) with $3+1$ parametrization (22), when a primary d-metric $\stackrel{\circ}{\mathrm{g}}=\left[\stackrel{\circ}{g}_{i}, \stackrel{\circ}{g}_{a}, \stackrel{\circ}{N_{b}^{j}}\right]$ defines a Kerr BH solution written in general coordinate fames which do not result in coordinate singularities of systems of PDEs of type (20), see details in references [7, 34, 43]. The QC structure generating function can be written in the form

$$
\bar{\Phi}=\Phi\left[{ }_{4} \bar{q}\right]=2 \sqrt{\left|\Lambda(\tau) \eta_{4}\left[{ }_{4} \bar{q}\right] \stackrel{\circ}{g}_{4}\right|},
$$

for $h_{4}(\tau)=\bar{q}\left(\tau, x^{i}, y^{3}\right)=\eta_{4}\left[{ }_{4} \bar{q}\right] \stackrel{\circ}{g}_{4}$, see (12). The effective volume form is computed

$$
d \overline{\mathcal{V}}\left[{ }_{h} \bar{q}, \eta_{4}\left[{ }_{4} \bar{q}\right], \bar{q}, \stackrel{\circ}{g}_{4}\right]=2 e^{\psi\left[{ }_{h} \bar{q}\right]} \sqrt{\frac{\left.\left|\eta_{4}\left[{ }_{4} \bar{q}\right] \stackrel{\circ}{g}_{4}\right| \eta_{4}\left[{ }_{4} \bar{q}\right]\right|^{\diamond} \mid}{\left.\left|\int d y^{3}{ }_{v} \bar{\Im}\right| \eta_{4}\left[{ }_{4} \bar{q}\right]\right|^{\diamond} \mid}} d x^{1} d x^{2}\left[d y^{3}+\frac{\partial_{i}\left(\int d y^{3}{ }_{v} \bar{\Im}\left|\eta_{4}\left[{ }_{4} \bar{q}\right]\right|^{\diamond}\right)}{{ }_{v} \bar{\Im}\left|\eta_{4}\left[{ }_{4} \bar{q}\right]\right|^{\diamond}} d x^{i}\right] d t
$$

which allows us to write down the thermodynamic generating function (26) in the form

$$
\overline{\mathcal{Z}}\left[{ }_{h} \bar{q}, \eta_{4}\left[{ }_{4} \bar{q}\right], \bar{q}, \stackrel{\circ}{g}_{4}\right]=\frac{1}{4 \pi^{2}} \int \tau^{-2} d \overline{\mathcal{V}} .
$$

Both the information on primary $\mathrm{BH}$ and $\mathrm{QC}$ data are encoded also in the thermodynamic variables (28) which for this type of nonholonomic stationary geometric flow evolution are computed

$$
\begin{aligned}
\overline{\mathcal{E}}\left[{ }_{h} \bar{q}, \eta_{4}\left[{ }_{4} \bar{q}\right], \bar{q}, \stackrel{\circ}{g}_{4}\right] & =-\frac{\tau^{2}}{4 \pi^{2}} \int\left(\left[{ }_{h} \Lambda(\tau)+\Lambda(\tau)\right]-\frac{2}{\tau}\right) \tau^{-2} d \overline{\mathcal{V}} \text { and } \\
\overline{\mathcal{S}}\left[{ }_{h} \bar{q}, \eta_{4}\left[{ }_{4} \bar{q}\right], \bar{q}, \stackrel{\circ}{g}_{4}\right] & =-\frac{1}{4 \pi^{2}} \int\left(\tau\left[{ }_{h} \Lambda(\tau)+\Lambda(\tau)\right]-2\right) \tau^{-2} d \overline{\mathcal{V}}
\end{aligned}
$$

and (in similar forms via $d \overline{\mathcal{V}}$ ). 


\subsubsection{Thermodynamics of small parametric stationary quasi-periodic BH deformations}

The d-metrics for such parametric solutions with linear decompositions on a small positive parameter $\varepsilon, 0 \leq \varepsilon \ll 1$, are described by a linear quadratic element (10) and a generating function (29) parameterized in the form

$$
\Phi\left[{ }_{4} \bar{q}, \stackrel{\circ}{g}_{4}\right] \simeq 2 \sqrt{\left|\Lambda(\tau) \stackrel{\circ}{g}_{4}\right|}\left(1-\frac{\varepsilon}{2} v\left[{ }_{4} \bar{q}\right]\right), \text { with } \bar{v}:=v\left[{ }_{4} \bar{q}\right],
$$

and a primary $\mathrm{BH}$ metric $\stackrel{\circ}{\mathrm{g}}=\left[\stackrel{\circ}{g}_{i}, \stackrel{\circ}{g}_{a}, \stackrel{\circ}{N}_{b}^{j}\right]$. Using the respective effective volume form

$$
d \overline{\mathcal{V}}_{[\varepsilon]}=2 e^{\psi\left[{ }_{h} i\right]}\left|\left(1-\frac{\varepsilon}{2} \bar{v}\right)\right| \sqrt{\frac{\left.\left|\stackrel{\circ}{g}_{4}\right| \bar{v}\right|^{\diamond} \mid}{\left.\left|\int d y^{3}{ }_{v} \bar{\Im}\right| \bar{v}\right|^{\diamond} \mid}} d x^{1} d x^{2}\left[d y^{3}+\frac{\partial_{i}\left(\int d y^{3}{ }_{v} \bar{\Im}|\bar{v}|^{\diamond}\right)}{{ }_{v} \bar{\Im}|\bar{v}|^{\diamond}} d x^{i}\right] d t
$$

we compute the corresponding thermodynamic generating function (26) and the canonical energy and entropy (28) for QC flow parametric deformations, respectively,

$$
\begin{aligned}
\overline{\mathcal{Z}}\left[{ }_{h} \bar{q}, \varepsilon \bar{v}, \bar{q}, \stackrel{\circ}{g}_{4}\right] & =\frac{1}{4 \pi^{2}} \int \tau^{-2} d \overline{\mathcal{V}}_{[\varepsilon]}, \text { resulting in } \\
\overline{\mathcal{E}}\left[{ }_{h} \bar{q}, \varepsilon \bar{v}, \bar{q}, \stackrel{\circ}{g}_{4}\right] & \left.=-\frac{\tau^{2}}{4 \pi^{2}} \int\left({ }_{h} \Lambda(\tau)+\Lambda(\tau)\right]-\frac{2}{\tau}\right) \tau^{-2} d \overline{\mathcal{V}}_{[\varepsilon]} \text { and } \\
\overline{\mathcal{S}}\left[{ }_{h} \bar{q}, \varepsilon \bar{v}, \bar{q}, \stackrel{\circ}{g}_{4}\right] & =-\frac{1}{4 \pi^{2}} \int\left(\tau\left[{ }_{h} \Lambda(\tau)+\Lambda(\tau)\right]-2\right) \tau^{-2} d \overline{\mathcal{V}}_{[\varepsilon]} .
\end{aligned}
$$

Finally, we emphasize that it is not possible to define and compute the Bekenstein-Hawking entropy for the exact and parametric QC nonholonomic deformation and/or $\mathrm{BH}$ solutions under geometric evolution or for certain self-similar configurations with Ricci solitons for a $\tau=\tau_{0}$. This is because gravitational and matter field configurations with QC are not characterized, in general, by certain horizon, duality of holographic conditions. BHs may evolve under geometric flows into another type of configurations with nonholonomically deformed horizons and additional degrees of freedom determined by generic off-diagonal terms of metrics and related N-connection coefficients describing locally anisotropic backgrounds and, for instance, polarization of constants etc. Such stationary quasi-periodic geometric flows are characterized by respective G. Perelman type thermodynamic variables as we computed in this section.

\section{Conclusion}

In this paper we elaborated on a geometric model of quasi-periodic Ricci flows as a proof of a geometric flow analog of the E. Verlinde conjecture [3, 24] that gravity is an emergent phenomenon linking to an entropic force which may explain dark matter properties. In our approach, the spacetime elasticity results from the thermodynamics of relativistic geometric flow evolution. Modified Einstein equations are derived from nonholonomic deformations [6, 7, 8, of $\mathrm{F}$ - and $\mathrm{W}$-entropies introduced by G. Perelman in order to prove the Poincaré-Thurston conjecture [4, 39, 40, 41, 42]. Based on our proposal, we shown that entropic geometric flows may result for certain well-defined conditions in stationary and quasi-periodic structures in MGTs and GR. This allows us to elaborate on dark matter and dark energy theories and to model structure formation in modern accelerating cosmology. We checked that for non-relativistic flows and zero nonholonomic torsion configurations the formulae coincide to those for the Hamilton-Perelman theory but reformulated for exact solutions parameterized with doubled $2+2,3+1$ fibration in pseudo-Riemannian geometry.

We note that Verlinde's conjecture implies the idea (present also in other thermodynamic, kinetic and diffusion like theories, see discussions and references in [46, 6]) that gravity is not a fundamental interaction but an emergent phenomenon. For instance, gravitational interactions may arise from the statistical behaviour of certain quantum / microscopic degrees of freedom encoded on a holographic screen. There are elaborated 
also models with quantum entanglement of local Rindler horizons and/or when gravity is a consequence of the "information associated with the positions of material bodies, etc. [17, 18, 20, 21, 27, 29]. For such constructions, there are necessary holographic screens, local Rindler horizons, BH or cosmological horizons, etc. It has not beem provided yet a rigorous mathematical formulation of Verlinde's conjecture and various theoretical models are under discussion and "criticism" [30, 31, 32]. In another turn, a respective relativistic generalization of the Poincaré-Thurston conjecture with canonical deformations of Perelman's W-entropy can be formulated in a rigorous mathematical physics form. Even this does not allow us to formulate and prove a Poincaré like hypothesis for four dimensional Lorentz manifolds (which is a very sophisticate task in modern mathematics), we can construct a self-consistent thermodynamic model for relativistic geometric [6, 7, 8] and information [9, 47, 33, 34, 35, flows. In such a geometric thermodynamic approach, the (modified) Einstein equations are derived as nonholonomic Ricci solitons (we cite 4, 38, 39, 40, 41, 42, for reviews of results on holonomic Ricci solitons). We do not need any assumptions on existence of holographic screens, nor any local Rindler horizons if we try to reconsider an analog of Verlinde's conjecture but connected to W-entropy and thermodynamics of relativistic Ricci flows.

The quaisi-periodic solutions for relativistic geometric flows, nonholonomic Ricci solitons and generalized gravitational field equations with quasi-periodic structure constructed and studied in this and our partner works [9, 33, 34, 35, 43, 45] are described by generic off-diagonal metrics and, in principle, by nonholonomically deformed non-Riemannian or (imposing additional constraints) pseudo-Riemannian connections. Such configurations are not characterized, in general, by entropy-area, holographic or duality conditions to certain conformal or gauge like models. Here we cite a recent work [48] with a different approach to entropy for a gauge gravity theory and our former results on noncommutative Ricci flows [5] with spectral triples following A. Connes approach and in connection to functional renormalizating group flow of quantum effective action for gravity [36, 37]. The end result is that we cannot rely on the thermodynamic models of such (modified) theories and exact or parametric solutions using only the concepts related to the Bekenstein-Hawking entropy. We argue that there is an alternative and more general approach when stationary and cosmological solutions in MGTs and GR can be derived and characterized using nonholonomic deformations of Perelman's W-entropy. Such constructions are similar to the well-known results on relativistic locally anisotropic thermodynamics and kinetics [6, 46]. In this paper, we provide explicit examples how to compute generalized Perelman thermodynamic variables for stationary quasi-periodic solutions describing in particular certain classes of general and small parametric black hole deformations.

Finally, we note that our approach provides new geometric methods and possible applications in the theory of quantum information flows, quantum systems with entanglement and emergent gravity and accelerating cosmology models simulated by classical and quantum computers. Such directions will be developed in our future works (see first results in [9, 47, 33, 34, 35] which seem to be more general and consist of a geometric/ quantum information flow alternative to approaches elaborated in [25, 26, 17, 18, 20, 21, 27, 19]).

Acknowledgments: This work belongs to a research program for a collaboration of S. Vacaru with the Yu. Fedkovych National Chernivtsi University in Ukraine. It develops former projects supported by IDEI, PN-II-ID-PCE-2011-3-0256, CERN and DAAD and a present adjunct position with California State University at Fresno, the USA. Authors are grateful to (co) editor and referee for very important criticism and requests which improved substantially the style of the paper.

\section{A $\quad 2+2$ and $3+1$ N-adapted Variables and Quasi-Periodic Structures}

We provide some important coefficient formulas and definitions on the geometry of noholonomic manifolds with duble $2+2$ and $3+1$ splitting and quasi-periodic structures, see details and proofs in [7, 43, 45, 47]. 


\section{A.1 Geometry of Lorentz manifolds with nonholonomic $2+2$ splitting}

We consider a 4-d Lorentzian manifold $V$ of signature $(+++-)$. Local coordinates on $V$ can be labeled for a conventional $2+2$ splitting when $u=(x, y)=\left\{u^{\alpha}=\left(x^{i}, y^{a}\right)\right\}$, for $\alpha=(i, a) ; \beta=(j, b)$, where $i, j, \ldots=1,2$ and $a, b, \ldots=3,4$, with $y^{4}=t$ being a time like coordinate. We can also consider a $3+1$ splitting with local coordinates $u^{\alpha}=\left(u^{\grave{\imath}}, t\right)$ for spacelike coordinates $u^{\grave{i}}=\left(x^{i}, y^{3}\right)$ when $\grave{i}, \grave{j}, \grave{k}, \ldots=1,2,3$. For such a spacetime manifold, a pseudo-Riemannian metric $\mathbf{g}$ can be parameterized in local coordinate and/or N-adapted form,

$$
\begin{aligned}
\mathbf{g}= & g_{\alpha \beta}\left(x^{i}, y^{a}\right) d u^{\alpha} \otimes d u^{\beta}, \text { for dual frame coordinate basis } d u^{\alpha} ; \\
= & \mathbf{g}_{\alpha \beta}(u) \mathbf{e}^{\alpha} \otimes \mathbf{e}^{\beta}=\mathbf{g}_{i}\left(x^{k}\right) d x^{i} \otimes d x^{i}+\mathbf{g}_{a}\left(x^{k}, y^{b}\right) \mathbf{e}^{a} \otimes \mathbf{e}^{b}, \\
& \text { for } \mathbf{e}^{\alpha}=\left(d x^{i}, \mathbf{e}^{a}=d y^{a}+N_{i}^{a}\left(u^{\gamma}\right) d x^{i}\right) \text { defining a N-adapted dual frame basis. }
\end{aligned}
$$

A $2+2$ splitting (A.2) is nonholonomic (equivalently, non-integrable, or anholonomic) with a co-basis $\mathbf{e}^{\alpha}=$ $\left(d x^{i}, \mathbf{e}^{a}\right)$ (A.3) dual to

$$
\mathbf{e}_{\alpha}=\left(\mathbf{e}_{i}, e_{a}\right)=\left(\mathbf{e}_{i}=\partial / \partial x^{i}-N_{i}^{a}(u) \partial / \partial y^{a}, e_{a}=\partial_{a}=\partial / \partial y^{a}\right),
$$

satisfying nonholonomy conditions

$$
\mathbf{e}_{[\alpha} \mathbf{e}_{\beta]}:=\mathbf{e}_{\alpha} \mathbf{e}_{\beta}-\mathbf{e}_{\beta} \mathbf{e}_{\alpha}=C_{\alpha \beta}^{\gamma}(u) \mathbf{e}_{\gamma}
$$

with anholonomy coefficients $C_{\alpha \beta}^{\gamma}=\left\{C_{i a}^{b}=\partial_{a} N_{i}^{b}, C_{j i}^{a}=\mathbf{e}_{j} N_{i}^{a}-\mathbf{e}_{i} N_{j}^{a}\right\}$. If such coefficients are nontrivial, the respective equivalent metric parameterizations (A.1) and (A.2) are generic off-diagonal. We can consider also frame transforms $\mathbf{e}^{\alpha}=\mathbf{e}_{\alpha^{\prime}}^{\alpha}(u) d u^{\alpha^{\prime}}$, when $g_{\alpha^{\prime} \beta^{\prime}}(u)=g_{\alpha \beta} \mathbf{e}_{\alpha^{\prime}}^{\alpha} \mathbf{e}_{\beta^{\prime}}$ performed in local coordinate or N-adapted forms.

Using standard formulas, we can define and compute both in abstract and coordinate forms the torsion, $\mathcal{T}$, the nonmetricity, $\mathcal{Q}$, and the curvature, $\mathcal{R}$, tensors for any distinguished connection, d-connection, $\mathbf{D}=(h D, v D)$, preserving the N-connection splitting under parallel maps along curves on spacetime $V$,

$$
\mathcal{T}(\mathbf{X}, \mathbf{Y}):=\mathbf{D}_{\mathbf{X}} \mathbf{Y}-\mathbf{D}_{\mathbf{Y}} \mathbf{X}-[\mathbf{X}, \mathbf{Y}], \mathcal{Q}(\mathbf{X}):=\mathbf{D}_{\mathbf{X}} \mathbf{g}, \mathcal{R}(\mathbf{X}, \mathbf{Y}):=\mathbf{D}_{\mathbf{X}} \mathbf{D}_{\mathbf{Y}}-\mathbf{D}_{\mathbf{Y}} \mathbf{D}_{\mathbf{X}}-\mathbf{D}_{[\mathbf{X}, \mathbf{Y}]} .
$$

There are used terms like distinguished tensor, d-tensor, and distinguished (geometric) object, d-object, (also d-metric, d-connection) etc. for geometric and physical values defined in N-adapted form. For instance, using $\mathrm{N}$-adapted coefficients of a d-connection, $\mathbf{D}=\left\{\boldsymbol{\Gamma}_{\alpha \beta}^{\gamma}=\left(L_{j k}^{i}, L_{b k}^{a}, C_{j c}^{i}, C_{b c}^{a}\right)\right\}$ (in some our works, we use hat symbols like $\widehat{\mathbf{D}}$ in order to state that certain geometric/ physical values are defined for a canonical d-connection), we can compute with respect to $\mathrm{N}$-adapted frames (A.4) and (A.3) the $\mathrm{N}$-adapted coefficients of respective d-tensors,

$\mathcal{T}=\left\{\mathbf{T}_{\alpha \beta}^{\gamma}=\left(T_{j k}^{i}, T_{j a}^{i}, T_{j i}^{a}, T_{b i}^{a}, T_{b c}^{a}\right)\right\}, \mathcal{Q}=\left\{\mathbf{Q}_{\alpha \beta}^{\gamma}\right\}, \mathcal{R}=\left\{\mathbf{R}_{\beta \gamma \delta}^{\alpha}=\left(R_{h j k}^{i}, R_{b j k}^{a}, R_{h j a}^{i}, R_{b j a}^{c}, R_{h b a}^{i}, R_{b e a}^{c}\right)\right\}$.

We omit cumbersome coefficient formulas which can be found in [7, 43].

The coefficients of the canonical d-connection $\mathbf{D}=\left\{\boldsymbol{\Gamma}_{\alpha \beta}^{\gamma}=\left(L_{j k}^{i}, L_{b k}^{a}, C_{j c}^{i}, C_{b c}^{a}\right)\right\}$ (1) can be computed following formulas

$$
\begin{aligned}
& L_{j k}^{i}=\frac{1}{2} g^{i r}\left(\mathbf{e}_{k} g_{j r}+\mathbf{e}_{j} g_{k r}-\mathbf{e}_{r} g_{j k}\right), C_{b c}^{a}=\frac{1}{2} g^{a d}\left(e_{c} g_{b d}+e_{b} g_{c d}-e_{d} g_{b c}\right), \\
& C_{j c}^{i}=\frac{1}{2} g^{i k} e_{c} g_{j k}, L_{b k}^{a}=e_{b}\left(N_{k}^{a}\right)+\frac{1}{2} g^{a c}\left(\mathbf{e}_{k} g_{b c}-g_{d c} e_{b} N_{k}^{d}-g_{d b} e_{c} N_{k}^{d}\right) .
\end{aligned}
$$

Similarly, we can compute in N-adapted form the coefficients of the distortion d-tensor, $\mathbf{Z}_{\alpha \beta}^{\gamma}=\boldsymbol{\Gamma}_{\alpha \beta}^{\gamma}-\Gamma_{\alpha \beta}^{\gamma}$, using (A.5) and LC-connection $\nabla=\left\{\Gamma_{\alpha \beta}^{\gamma}\right\}$. The nontrivial d-torsion coefficients $\mathbf{T}_{\alpha \beta}^{\gamma}$ are

$$
T_{j k}^{i}=L_{j k}^{i}-L_{k j}^{i}, T_{j a}^{i}=C_{j b}^{i}, T_{j i}^{a}=-\Omega_{j i}^{a}, T_{a j}^{c}=L_{a j}^{c}-e_{a}\left(N_{j}^{c}\right), T_{b c}^{a}=C_{b c}^{a}-C_{c b}^{a} .
$$


The d-torsion coefficients (A.6) vanish if in $\mathrm{N}$-adapted form

$$
L_{a j}^{c}=e_{a}\left(N_{j}^{c}\right), C_{j b}^{i}=0, \Omega_{j i}^{a}=0 .
$$

Using formulas (A.5) and (A.6), we can compute the coefficients of the Riemann d-tensor, $\mathbf{R}_{\beta \gamma \delta}^{\alpha}$, and the Ricci d-tensor, $\mathbf{R}_{\alpha \beta}$.

\section{A.2 Nonholonomic distributions with quasiperiodic and/or pattern forming structures}

We shall work with necessary smooth classes of functions $q=q\left(x^{i}, y^{3}\right)$, for space like distributions, and $\bar{q}=\bar{q}\left(x^{i}, y^{4}=t\right)$, for locally anisotropic cosmological configurations. Such $q$ and/or $\bar{q}$ can be considered as generating functions and/or (effective) sources for different models of quasiperiodic and/or pattern forming spacetime structures.

A metric (A.1) and/or d-metric (A.2) can be re-written in a form with nonholonomic $3+1$ splitting,

$$
\begin{aligned}
\mathbf{g} & =b_{i}\left(x^{k}\right) d x^{i} \otimes d x^{i}+b_{3}\left(x^{k}, y^{3}, t\right) \mathbf{e}^{3} \otimes \mathbf{e}^{3}-\breve{N}^{2}\left(x^{k}, y^{3}, t\right) \mathbf{e}^{4} \otimes \mathbf{e}^{4}, \\
\mathbf{e}^{3} & =d y^{3}+N_{i}^{3}\left(x^{k}, y^{3}, t\right) d x^{i}, \quad \mathbf{e}^{4}=d t+N_{i}^{4}\left(x^{k}, y^{3}, t\right) d x^{i} .
\end{aligned}
$$

A 4- $\mathrm{d}$ metric $\mathbf{g}$ can be considered as an extension of a $3-\mathrm{d}$ metric $\mathbf{b}=\left\{b_{i j}=\operatorname{diag}\left(b_{\grave{\imath}}\right)=\left(b_{i}, b_{3}\right)\right\}$ on a family of 3-d hypersurfaces $\widehat{\Xi}_{t}$ parameterized by coordinate parameter $t$, when $b_{3}=h_{3}$ and $\breve{N}^{2}(u)=-h_{4}$ is defined by a lapse function $\breve{N}(u)$. Such a decomposition results in a representation $\mathbf{D}=\left({ }^{3} \mathbf{D},{ }^{t} \mathbf{D}\right)$. The operator where ${ }^{3} \mathbf{D}$ defines the action of the canonical d-connection covariant derivative on space like coefficients. The operator ${ }^{t} \mathbf{D}$ of time like coefficients. Similarly, the LC covariant derivative operator splits as $\nabla=\left({ }^{3} \nabla,{ }^{t} \nabla\right)$; the action on a scalar field $q(u)$ can be parameterized via frame/ coordinate transforms as $\nabla q=\left({ }^{3} \nabla q,{ }^{t} \nabla q=\partial_{t} q=q^{*}\right)$.

Models with tree-waves interactions, 3WIs, for many pattern-forming systems can be elaborated as in condensed matter physics. Modern cosmological data show a very complex web like quasiperiodic and/or aperiodic like structure formation, geometric anisotropic evolution and nonlinear gravitational and matter field interactions. We can apply mathematical methods for modeling quasi-crystal matter or (super) galactic clusters and 3-d distributions of dark energy and dark matter.

A prime pattern-forming field can be taken in the form

$$
\bar{q}\left(x^{i}, t\right)=\sum_{l=1,\left|\mathbf{k}_{l}\right|=1}^{\infty} z_{l}(t) e^{i \mathbf{k}_{l} \cdot \mathbf{u}}+\sum_{l=1,\left|\mathbf{c}_{l}\right|=c} v_{l}(t) e^{i \mathbf{c}_{l} \cdot \mathbf{u}}+\text { higher order terms }
$$

defining in flat spaces 3WIs involving two comparable wavelengths. We consider systems with two wave numbers $k=1$ and $k=c$ (this constant $c$ should be not confused with the speed of light) for $0<c<1$. Constructions with 3 WIs are modelled in two forms:

1. using two waves (with wave number 1 , on the outer circle) interact nonlinearly with a wave on the inner circle, with wave number $c$ (for instance, wave vectors configurations $\mathbf{k}_{1}$ and $\mathbf{k}_{2}$ interact with $\left.\mathbf{c}_{1}=\mathbf{k}_{1}+\mathbf{k}_{2}\right)$

2. considering for $1 / 2<c<1$ two waves on the inner circle interact with another wave on the outer circle; we can consider wave vectors configurations $\mathbf{c}_{2}$ and $\mathbf{c}_{3}$ interact with $\mathbf{k}_{1}=\mathbf{c}_{2}+\mathbf{c}_{3}$.

For a decomposition (A.8), we can parameterize the coefficients when there are modelled two types of 3 WIs. There are considered (in the case 1 ) a triad of wave vectors $\mathbf{k}_{1}, \mathbf{k}_{2}$ and $\mathbf{c}_{1}=\mathbf{k}_{1}+\mathbf{k}_{2}$, when amplitudes are subjected to the conditions:

$z_{1}^{*}=\mu z_{1}+Q_{z v} \bar{z}_{2} v_{1}+$ cubic terms, $z_{2}^{*}=\mu z_{2}+Q_{z v} \bar{z}_{1} v_{1}+$ cubic terms, and $v_{1}^{*}=\xi z_{1}+Q_{z z} z_{1} z_{2}+$ cubic terms. 
In the case 2 with wave vectors $\mathbf{c}_{2}, \mathbf{c}_{3}$ and $\mathbf{k}_{1}=\mathbf{c}_{2}+\mathbf{c}_{3}$ and equations for amplitudes,

$v_{2}^{*}=\xi v_{2}+Q_{v z} \bar{v}_{2} z_{1}+$ cubic terms, $v_{3}^{*}=\xi v_{3}+Q_{v z} \bar{v}_{1} z_{1}+$ cubic terms, and $z_{1}^{*}=\mu z_{1}+Q_{z z} v_{2} v_{3}+$ cubic terms.

Respectively, the coefficients $\mu$ and $\xi$ describe the growth rates of amplitudes corresponding to wave numbers 1 and $c$; when, for instance, $Q_{z v}$ and $Q_{z z}$ are quadratic elements.

We can consider more general nonlinear deformations of prime waves (A.8), $\bar{q}\left(x^{i}, t\right) \rightarrow P \bar{q}\left(x^{i}, t\right)$, when the target field ${ }^{P} \bar{q}=\bar{q}$ describes pattern forming configurations as solutions of nonlinear PDE,

$$
\bar{q}^{*}=\mathcal{L} \bar{q}+{ }^{1} Q \bar{q}^{2}+{ }^{2} Q\left({ }^{3} \mathbf{D}_{\grave{i}}{ }^{3} \mathbf{D}^{\grave{q}} \bar{q}\right)+{ }^{3} Q\left|\left({ }^{3} \mathbf{D}_{i} \bar{q}\right)\right|^{2}-\bar{q}^{3},
$$

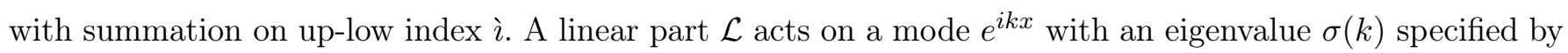
$\sigma(1)=\mu$ and $\sigma(c)=\xi$. This controls growth rates of the modes of interest; with $d \sigma / d x=0$. For $k=1$ and $k=c$, and $\sigma(0)=\sigma_{0}<0$, we control the depths of minimum between $k=1$ and $k=c$. If there are chosen even functions $\sigma$ of $k$, we can consider a 4th order polynomial on $k^{2}$,

$$
\begin{aligned}
& \sigma(k)=\frac{[\mu A(k)+\xi B(k)] k^{2}}{\left(1-c^{2}\right)^{3} c^{4}}+\frac{\sigma_{0}}{c^{4}}\left(1-k^{2}\right)\left(c^{2}-k^{2}\right)^{2}, \text { where } \\
& A(k)=\left[\left(c^{2}-3\right) k^{2}-2 c^{2}+4\right]\left(k^{2}-c^{2}\right)^{2} c^{4} \text { and } B(k)=\left[\left(3 c^{2}-1\right) k^{2}+2 c^{2}-4 c^{4}\right]\left(k^{2}-1\right)^{2} .
\end{aligned}
$$

To elaborate on cosmological theories we can consider scalar fields potentials $V(\varphi)$ modified by effective quasicrystal structures, $\varphi \rightarrow \varphi=\varphi_{0}+\psi$, where $\psi\left(x^{i}, y^{3}, t\right)$ with (quasi) crystal like phases described by periodic or quasi-periodic modulations. We can construct models of 3-d non-relativistic dynamics which determined by Laplace like operators ${ }^{3} \Delta=\left({ }^{3} \nabla\right)^{2}$, or ${ }^{b} \widehat{\Delta}$ (the left label 3 emphasizes that such an operator is for a 3-d hypersurface). Writing $\psi$ instead of $\bar{q}$ in order to distinguish such QC structures (generated both by gravitational and matter field with two length scales) from the class of models discussed above.

For a $\mathrm{N}$-adapted $3+1$ splitting, the equations for a local minimum conserving dynamics,

$$
\partial_{t} \psi={ }^{3} \triangle\left[\frac{\delta F[\psi]}{\delta \psi}\right] \text {, or in a nonholonomic variant } \partial_{t} \psi={ }^{b} \widehat{\Delta}\left[\frac{\delta F[\psi]}{\delta \psi}\right],
$$

with two length scales $l_{\underline{i}}=2 \pi / k_{\underline{i}}$, for $\underline{i}=1,2$. Local diffusion processes are determined by a free energy functional

$$
\begin{aligned}
F[\psi] & =\int \sqrt{\left|{ }^{3} g\right|} d x^{1} d x^{2} d y^{3}\left[\frac{1}{2} \psi\left\{-\epsilon+\prod_{\underline{i}=1,2}\left(k_{\underline{i}}^{2}+{ }^{3} \triangle\right)^{2}\right\} \psi+\frac{1}{4} \psi^{4}\right], \\
\text { or }{ }^{b} F[\psi] & =\int \sqrt{\left|{ }^{3} g\right|} d x^{1} d x^{2} d y^{3}\left[\frac{1}{2} \psi\left\{-\epsilon+\prod_{\underline{i}=1,2}\left(k_{\underline{i}}^{2}+{ }^{b} \widehat{\Delta}\right)^{2}\right\} \psi+\frac{1}{4} \psi^{4}\right],
\end{aligned}
$$

where $\left|{ }^{3} g\right|$ is the determinant of the 3 -d space metric and $\epsilon$ is a constant. Finally, we note that the functional ${ }^{b} F[\psi]$ is defined by a nonholonomic deformation of the Laplace operator, ${ }^{3} \triangle \rightarrow{ }^{b} \widehat{\Delta}$, resulting in a nonholonomic distortion of $F[\psi]$.

\section{References}

[1] T. Jacobson, Thermodynamics of space-time: The Einstein equation of state, Phys. Rev. Lett. 75 (1995) 1260-1263, arXiv: gr-qc/9504004

[2] T. Padmanabhan, Thermodynamical aspects of gravity: new insights,Rept. Prog. Phys. 73 (2010) 046901; arXiv: 0911.5005 
[3] E. P. Verlinde, On the origin of gravity and the laws of Newton, JHEP 1104 (2011) 029; arXiv: 1001.0785

[4] G. Perelman, The entropy formula for the Ricci flow and its geometric applications, arXiv: math. DG/0211159

[5] S. Vacaru, Spectral functionals, nonholonomic Dirac operators, and noncommutative Ricci flows, J. Math. Phys. 50 (2009) 073503; arXiv: 0806.3814 [math-ph]

[6] V. Ruchin, O. Vacaru and S. Vacaru, Perelman's W-entropy and Statistical and Relativistic Thermodynamic Description of Gravitational Fields, Eur. Phys. J. C 77 (2017) 184; arXiv: 1312.2580

[7] T. Gheorghiu, V. Ruchin, O. Vacaru and S. Vacaru, Geometric flows and Perelman's thermodynamics for black ellipsoids in R2 and Einstein gravity theories; Annals of Physics, NY, 369 (2016) 1-35; arXiv: 1602.08512

[8] S. Rajpoot and S. Vacaru, On supersymmetric geometric flows and R2 inflation from scale invariant supergravity, Annals of Physics, NY, 384 (2017) 20 -60; arXiv: 1606.06884

[9] S. Vacaru and L. Bubuianu, Classical and quantum geometric information flows and entanglement of relativistic mechanical systems, Quantum Inf. Process, QING 18 (2019) 376; arXiv: 1905.13015

[10] J. D. Bekenstein, Black holes and the second law, Nuovo Cimento Letters 4 (1972) 737-740

[11] J. D. Bekenstein, Black holes and entropy, Phys. Rev. D 7 (1973) 2333-2346

[12] J. M. Bardeen, B. Carter and S. W. Hawking, The four laws of black hole mechanics, Commun. Math. Phys. 31 (1973) 161

[13] S. W. Hawking, Particle creation by black holes, Commun. Math. Phys. 43 (1975) 199-220

[14] A. Strominger and C. Vafa, Microscopic origin of the Bekenstein-Hawking entropy, Phys. Lett. B 379 (1996) 99; arXiv: hep-th/9601029

[15] J. M. Maldacena, The large N limit of superconformal field theories and supergavity, Adv. Theor. Math. Phys. 2 (1998) 231; arXiv: hep-th/9711200

[16] A. Ashtekar and B. Krishnan, Isolated and dynamical horizons and their application, Living Rev. Rel. 7 (2004) 10; arXiv: gr-qc/0407042

[17] S. Ryu and T. Takayanagi, Holographic derivation of entanglement entropy from AdS/CFT, Phys. Rev. Lett. 96 (2006) 181602; arXiv: hep-th/0603001

[18] M. Van Raamsdonk, Building up spacetime with quantum entanglement, Gen. Rel. Grav. 42 (2010) 2323 [Int. J. Mod. Phys. D 19 (2010) 2429]; arXiv: 1005.3035

[19] S. Lloyd, The quantum geometric limit, arXiv: 1206.6559

[20] T. Faulkner, M. Guica, T. Harman, R. C. Myers and M. Van Raamsdonk, Gravitation from entanglement and holographic CFTs, JHEP 1403 (2015) 051; arXiv: 1312.7856

[21] B. Swingle, Entanglement renormalization and holography, Phys. Rev. D 86 (2012) 065007; arXiv: 0905.1317

[22] T. Jacobson, Entanglement equilibrium and the Einstein equation, Phys. Rev. Lett. 116 (2016) 201101; arXiv: 1505.04753 
[23] F. Pastawski, B. Yoshida, D. Harlow and J. Preskill, Holographic quantum error-correcting codes: Toy models for the bulk/boundary correspondence, JHEP, 1506 (2015) 149; arXiv:1503.06237

[24] E. Verlinde, Emergent gravity and the dark universe, SciPost Phys. 2 (2017) no.3, 016; arXiv: 1611.02269

[25] E. Witten, A mini-introduction to information theory, arXiv: 1805.11965

[26] J. Preskill, lecture notes, http://www.theory.caltech.edu/ preskill/ph219/index.html\#lecture

[27] H. Casini, M. Huerta and R. C. Myers, Towards a derivation of holographic entanglement entropy, JHEP 1105 (2011) 036; arXiv:1102.0440

[28] A. Lewkowycz and J. Maldacena, Generalized gravitational entropy, JHEP 1308 (2013) 090; arXiv: 1304.4926

[29] S. N. Solodukhin, Entanglement entropy of black holes, Living Rev. Rel. 14 (2011) 8, arXiv: 1104.3712

[30] S. Hossenfelder, Covariant version of Verlinde's emergent gravity, Phys. Rev. D95 (2017) 124018; arXiv. 1703.01415

[31] De-Chang Dai and D. Stojkovic, A note on covariant version of Verlinde's emergent gravity' Phys. Rev. D96 (2017) 108501; arXiv: 1706.07584

[32] De-Chang Dai and D. Stojkovic, Inconsistencies in Verlinde's emergent gravity, JHEP 1711 (2017) 007; arXiv: 1710.00946

[33] S. Vacaru, E. Veliev and L. Bubuianu, Exact solutions in emergent gravity theories and generalized G. Perelman entropy for geometric flows, arXiv: 1904.05149v3

[34] Iuliana Bubuianu, S. Vacaru and E. V. Veliev, Quantum geometric information flows and relativistic generalizations of Grigory Perelman thermodynamics for nonholonomic Einstein systems with black holes and stationary solitonic hierarchies, an extension of S. Vacaru and Iuliana Bubuianu, Quantum geometric information flows and emergent gravity, arXiv: 1907.03541v3

[35] Iuliana Bubuianu, S. Vacaru and E. V. Veliev, Kaluza-Klein gravity \& cosmology emerging from G. Perelman's entropy functionals and quantum geometric information flows, arXiv: 1907.05847v3

[36] D. Friedan, Nonlinear models in $2+\varepsilon$ dimensions, Phys. Rev. Lett. 45 (1980) $1057-1060$

[37] D. Friedan, Ricci flows. Nonlinear models in 2+ $\varepsilon$ dimensions, Annals of Physics, NY, 163 (1985) 318-419

[38] R. S. Hamilton, Three-manifolds with postive Ricci curvature, J. Diff. Geom. 17 (1982) 255-306

[39] H. -D. Cao and H. -P. Zhu, A complete proof of the Poincaré and geometrization conjectures - application of the Hamilton-Perelman theory of the Ricci flow, Asian J. Math. 10 (2006) 165-495

[40] J. W. Morgan and G. Tian, Ricci flow and the Poincaré conjecture, AMS, Clay Math.Monog. v.3 (2007)

[41] B. Kleiner and J. Lott, Notes on Perelman's papers, Geometry \& Topology 12 (2008) 2587-2855

[42] Huai-Dong Cao, Recent progress on Ricci solitons, Recent advances in geometric analysis, Adv. Lect. Math. (ALM) 11 (2009) 1-38, arXiv: 0908.2006 [math.DG]

[43] L. Bubuianu and S. Vacaru, Deforming black hole and cosmological solutions by quasiperiodic and/or pattern forming structures in modified and Einstein gravity, Eur. Phys. J. C78 (2018) 393; arXiv: 1706.02584 
[44] S. Nojiri, S. D. Odintsov and V. K. Oikonomou, Modified gravity theories in nutshell: inflation, bounce and late-time evolution, Phys. Rept. 692 (2017) 1-104; arXiv: 1705.11098

[45] S. Vacaru, Space-time quasicrystal structures and inflationary and late time evolution dynamics in accelerating cosmology, Class. Quant. Grav. 35 (2018) 245009; arXiv: 1803.04810

[46] S. Vacaru, Locally anisotropic kinetic processes and thermodynamics in curved spaces, Ann. Phys. (N.Y.) 290 (2001) 83-123; arXiv: gr-qc/0001060

[47] S. Vacaru, Geometric information flows and G. Perelman entropy for relativistic classical and quantum mechanical systems, Eur. Phys. J. C 80 (2020) 639; arXiv: 1905.12399

[48] M. Blagojević and B. Cvetković, Entropy in Poincaré gauge theory: Kerr-AdS soluiton, arXiv: 2007.10721 